\title{
Synchrotron x-ray-diffraction study of the structure and growth of Xe films adsorbed on the $\operatorname{Ag}(111)$ surface
}

\author{
Pengcheng Dai, ${ }^{*}$ Z. Wu ${ }^{\dagger}$ T. Angot, ${ }^{\ddagger}$ S.-K. Wang, ${ }^{\S}$ and H. Taub ${ }^{\|}$ \\ Department of Physics and Astronomy, University of Missouri-Columbia, Columbia, Missouri 65211 \\ S. N. Ehrlich \\ School of Materials Engineering, Purdue University, West Lafayette, Indiana 47907
}

(Received 22 June 1998)

\begin{abstract}
Synchrotron x-ray scattering has been used to investigate the structure and growth of perhaps the simplest of all films: xenon physisorbed on the $\operatorname{Ag}(111)$ surface. High-resolution x-ray scans of the in-plane structure and lower-resolution scans (specular and nonspecular) of the out-of-plane order were performed. The Xe films were prepared under both quasiequilibrium and kinetic growth conditions, and have fewer structural defects than those investigated previously by others on graphite substrates. Under quasiequilibrium conditions, the bulk $\mathrm{Xe}$-Xe spacing is reached at monolayer completion, and the monolayer and bilayer lattice constants at coexistence are inferred equal to within $0.005 \AA$, consistent with theoretical calculations. The Xe/vacuum interface profile for a complete monolayer and bilayer grown at quasiequilibrium is found to be sharper than for kinetically grown films. At coverages above two layers, diffraction scans along the $\mathrm{Xe}(01 l)$ rod for quasiequilibrated films are consistent with the presence of two domains having predominantly an $A B C$ stacking sequence and rotated $60^{\circ}$ with respect to each other about the surface normal. Annealing of these films alters neither the population of the two domains nor the fraction of $A B A$ stacking faults. The thickest film grown under quasiequilibrium conditions exceeds $220 \AA$ (resolution limited). Under kinetic growth conditions, $\mathrm{x}$-ray intensity oscillations at the Xe anti-Bragg position of the specular rod are observed as a function of time, indicating nearly layer-by-layer growth. Up to four complete oscillations corresponding to a film of eight layers have been observed before the intensity is damped out; the number of oscillations is found to depend on the substrate temperature, the growth rate, and the quality of the $\operatorname{Ag}(111)$ substrate. The specular reflectivity from kinetically grown films at nominal coverages of three and four layers has been analyzed using a Gaussian model which gives a film thickness standard deviation of 0.5 and 1.0 layers, respectively. Diffraction scans along the $\mathrm{Xe}(01 l)$ rod of these films indicate a larger fraction of $A B A$ stacking faults than found for thicker films. These results demonstrate the difficulty of kinetically growing Xe films thicker than two layers which have an ideal slab geometry. [S0163-1829(99)03723-6]
\end{abstract}

\section{INTRODUCTION}

Heavier rare gases physically adsorbed on close-packed single-crystal metal surfaces are usually considered to be the simplest of all films for theoretical study. ${ }^{1,2}$ Since the zeropoint motion of these atoms can be neglected, the adatomadatom and adatom-substrate interactions can be represented relatively easily and accurately by analytical expressions. ${ }^{1}$ For this reason, rare-gas films are expected to provide a benchmark for the degree of understanding which one can hope to achieve for other physisorbed films.

These expectations have been largely realized for $\mathrm{Xe}, \mathrm{Kr}$, and $\mathrm{Ar}$ monolayers adsorbed on a $\operatorname{Ag}(111)$ surface. The delocalized conduction electrons of the silver yield a smoother effective surface than found on insulating substrates. ${ }^{1}$ Comprehensive experimental $^{3,4}$ and theoretical studies ${ }^{5-7}$ have provided a rather detailed understanding of the monolayer phase diagram, lattice spacings, latent heats, and heats of adsorption. In contrast, it has been somewhat more difficult to achieve the same degree of understanding for these monolayers adsorbed on the basal-plane surfaces of graphite, another widely used substrate, where registry effects due to the lateral variation (corrugation) in the adatom-substrate binding energy are larger. ${ }^{1}$ Indeed, corrugation effects result in topological differences in the rare-gas monolayer phase diagrams on graphite. ${ }^{8}$

Above a monolayer thickness, not only do calculations of structural and thermodynamic properties of rare-gas films increase in complexity, but the difficulty of growing highquality films increases as well. The low-energy electron diffraction (LEED) experiments of Unguris et al. ${ }^{4}$ showed that it is very difficult to achieve ideal layer-by-layer growth for film thicknesses above two layers under quasiequilibrium conditions. In these experiments, the attenuation of a $\mathrm{Ag}$ diffraction peak is monitored as a function of the substrate temperature while dosing with a constant flux of gas. Only two distinct steplike decreases in intensity were observed upon lowering the temperature, corresponding to monolayer and bilayer condensation. The binding energies of higher layers are so closely spaced that, with experimentally achievable control of temperature and pressure, one cannot resolve individual layer condensations above two layers by this LEED isobar method. ${ }^{4}$

In the case of growth far from equilibrium or "kinetic" growth, experimental investigations by helium-atom scattering, ${ }^{9}$ photoemission, ${ }^{10}$ thermal desorption, ${ }^{9,10}$ and surface-plasmon-resonance ${ }^{11}$ techniques provide evidence of layer-by-layer growth at least up to three layers. The situa- 
tion has been reviewed by Gibson and Sibener in their second paper of Ref. 9. Their thermal-desorption spectra showed distinct desorption temperatures for the first three layers of gas with each higher layer desorbing at a lower temperature. In addition, they found that dosing a completed trilayer with a small quantity of gas resulted in a peak or shoulder appearing on the low-temperature side of the trilayer desorption peak consistent with the third layer being more strongly bound than the fourth. Gibson and Sibener noted that the narrow angular widths of the elastic features observed in their He-atom-scattering experiments provide qualitative evidence of a sharp film/vacuum interface. They also cited the distinct surface vibrational spectra observed by inelastic He-atom scattering for one, two, and three layers of the rare gases on the $\operatorname{Ag}(111)$ surface as evidence of layerby-layer growth. ${ }^{9}$

Gibson and Sibener's thermal-desorption experiments were consistent with earlier results of Ref. 10. The latter found the area under the thermal desorption peaks from the saturated first and second layer of $\mathrm{Xe} / \mathrm{Ag}(111)$ to be the same (within 5\%) and hence the same particle density for both layers to within this uncertainty. From their x-ray photoelectron spectroscopy experiments, the authors of Ref. 10 concluded that layer-by-layer growth occurred up to three layers. ${ }^{10}$ Also, a weak knee in the photoemission intensity from the $\mathrm{Xe}\left(3 d_{5 / 2}\right)$ level suggested adsorption of a fourth $\mathrm{Xe}$ layer on $\operatorname{Ag}(111)$.

The surface-plasmon-resonance experiments of Qian and Bretz ${ }^{11}$ offered additional evidence of layerwise growth in this system. They observed steplike features up to about four layers in the diffuse light intensity scattered from Xe films adsorbed on polycrystalline Ag substrates.

It should be emphasized that the experiments described above have not provided a quantitative measure of the roughness of the film/vacuum interface. Such microscopic structural properties of the films are best probed by diffraction techniques. In the case of multilayer rare-gas films, most of the previous diffraction studies have been performed on graphite substrates. Neutron-diffraction experiments ${ }^{12}$ require exfoliated substrates to achieve the requisite high surface areas. Only quasiequilibrium growth is possible with these substrates, and capillary condensation effects ${ }^{2,12}$ have tended to obscure the multilayer structure of the film. In addition, profile analysis of the powder diffraction patterns lacks the sensitivity to detect fractions of a layer. For these reasons, it has not been possible to characterize the density profile of the film/vacuum interface with sufficient precision to confirm layer-by-layer growth above film thicknesses of two layers.

Rare-gas films have been investigated on single-crystal graphite substrates in $\mathrm{x}$-ray-diffraction experiments utilizing a rotating anode source. ${ }^{13}$ For Xe films on the (0001) graphite surface, a high degree of stacking disorder prevented detailed modeling. Also, the use of a vermicular graphite as a gas ballast to measure and maintain a precise Xe coverage failed to eliminate the possibility of capillary condensation effects.

In addition to these these experimental investigations, there have also been theoretical studies of the structural and thermodynamic properties of rare-gas multilayers on the $\operatorname{Ag}(111)$ surface. Wei and Bruch $^{14}$ considered the coexist- ence of monolayer and bilayer solid films in order to compare results with the LEED isobar experiments of Unguris et al. ${ }^{4}$ For $\mathrm{Xe} / \mathrm{Ag}(111)$, they found a lattice constant discontinuity at the monolayer-bilayer transition of less than 0.005 $\AA$ consistent with the LEED experiments. ${ }^{4}$ However, their calculations gave a significant difference between the film lattice constant at the transition, referred to as the monolayer limit of compression, and the measured lattice constant of solid bulk Xe. This result conflicted with the LEED experiments, ${ }^{4}$ which found the bulk and film lattice constants at the limit of compression to be indistinguishable within an accuracy of $0.01 \AA$.

In a subsequent paper, Bruch and $\mathrm{Ni}^{15}$ reported model calculations for Xe trilayers on the $\operatorname{Ag}(111)$ surface. They found these trilayers to be more stable than the formation of bulk $\mathrm{Xe}$ as well as the Xe trilayer on the graphite (0001) surface. Both $A B A$ stacking of a hexagonal-close-packed (hcp) structure, and $A B C$ stacking of a face-centered-cubic structure were considered. Although calculated energy differences were small $\left(<0.5 \mathrm{Katom}^{-1}\right), A B A$ stacking was more favorable.

The stability of Xe trilayers on the $\operatorname{Ag}(111)$ surface relative to the bulk phase is in accord with the experiments reviewed above. ${ }^{9-11}$ However, there has been little experimental investigation of the stacking sequence of Xe films on this substrate. Neutron-diffraction experiments on ${ }^{36} \mathrm{Ar}$ multilayer films adsorbed on polycrystalline graphite found some evidence for coexistence of $A B A$ and $A B C$ stacking, with the $A B C$ sequence dominating as the film thickens. ${ }^{12}$ But, as noted above, these experiments were hampered by capillary condensation.

Thus, despite the remarkable simplicity of these prototypical films and a large number of experiments, basic questions concerning their structure remain unresolved. These include the following: (1) To what extent can the films be grown layer-by-layer? (2) Above bilayer thickness, are successive layers identical in structure and mutually commensurate? (3) What is the stacking sequence of the layers and are there stacking faults? (4) What is the density profile at the film/vacuum interface. (5) What is the ultimate film thickness?

From the foregoing discussion, it can be seen that addressing these questions experimentally requires not only growing high-quality multilayer rare-gas films but doing so under conditions where the most structurally sensitive probes can be applied. One of these probes is synchrotron x-ray scattering, which has developed rapidly in recent years as a technique for in situ monitoring of surface structure during film growth. The method has been applied to layer-by-layer growth at semiconductor ${ }^{16,17}$ and metal surface, ${ }^{18}$ as well as at a metal-semiconductor interface. ${ }^{19}$ These experiments have exploited the extreme brilliance of synchrotron $\mathrm{x}$-ray sources to obtain submonolayer sensitivity, as well as high resolution in reciprocal space for a precise determination of structural parameters. Unlike more surface-sensitive probes, the penetrability of $\mathrm{x}$-rays allows one to follow the structural evolution of the surface region from monolayer thickness to bulk. $^{20}$

Common to these surface $\mathrm{x}$-ray experiments ${ }^{16-19}$ has been the demonstration of layerwise growth by the observation of intensity oscillations in the specularly reflected $\mathrm{x}$-ray beam 
(incident and reflected angles equal) as a function of time. The amplitude of these oscillations is a sensitive measure of structural perfection in the surface region. While in this respect they are similar to intensity oscillations routinely observed by reflection high-energy electron diffraction during film growth by molecular-beam epitaxy, ${ }^{21}$ the reflected x-ray intensity permits a kinematic analysis allowing a more detailed modeling of surface structure and morphology.

The brilliance of synchrotron x-ray sources also allows measurement of the wave vector $\left(Q_{\perp}\right)$ dependence of the specular reflectivity from adsorbed films at coverages as low as a monolayer. ${ }^{22}$ These specular scans measure the Fourier transform of the atomic density profile perpendicular to the surface, and can be analyzed kinematically to infer the number of atomic layers in the film, the density of atoms within a layer, the height of the first film layer above the substrate, and the separation between film layers, i.e., a quantitative characterization of the film roughness. While specular Heatom scattering has been reported from rare-gas films on the $\operatorname{Ag}(111)$ substrate, ${ }^{9}$ its wave-vector dependence was apparently not measured resulting in only a qualitative measure of the film roughness.

In a specular reflectivity measurement, there is no wavevector transfer parallel to the surface $\left(Q_{\|}=0\right)$, so that spatial correlations within a film layer are not probed. However, nonspecular $\mathrm{x}$-ray scans along Bragg rods of the film ${ }^{20}$ are sensitive to these lateral correlations and, in principle, can be used to determine the stacking sequence in the film. As we have seen, it is of particular interest in rare-gas films to determine whether the films actually have the $A B C$ stacking sequence expected for the bulk fcc structure or whether there might be stacking faults corresponding to the $A B A$ stacking of an hep structure. Again, these issues are difficult to address with LEED and He-atom scattering which probe only the topmost layers of the film.

The intrinsic collimation of a synchrotron x-ray beam as well as its intensity permit higher-resolution scans in reciprocal space than is possible by LEED or He-atom scattering. This capability can be used to obtain extremely precise measurements of the intralayer lattice constants of the film as its thickness increases. It is of interest to exploit this precision in measurements on films as structurally simple as the rare gases. In particular, we shall see that it allows a more sensitive measure of the lattice constant discontinuity at monolayer-bilayer coexistence.

Unfortunately, synchrotron x-ray-diffraction studies of the structure and growth of physisorbed films have lagged behind those on semiconductor and metal surfaces. The reasons for this are in part the technical difficulties of achieving a wide temperature range- - high enough to clean the singlecrystal metal substrate while sufficiently low to adsorb the weakly bound film. In addition, there is need for relatively rapid independent characterization of the film and substrate such as by in situ LEED. These capabilities must be achieved with an ultrahigh-vacuum chamber compatible with the requisite $\mathrm{x}$-ray diffractometer.

To exploit the advantages of synchrotron x-ray diffraction for surface structure determination and to overcome some of the problems encountered in previous x-ray studies of physisorbed films, we have constructed an ultrahigh-vacuum chamber which is particularly well suited for structural analysis of films physisorbed on single-crystal substrates. ${ }^{23,24}$ The chamber has both a wide temperature range and an in situ LEED capability, and mounts on a standard four-circle x-ray diffractometer. Our use of a single-crystal substrate without a polycrystalline ballast for coverage determination eliminates capillary condensation effects, ${ }^{12}$ and allows films to be grown with a higher degree of structural perfection than previously achieved. ${ }^{13}$

In this paper, we report results obtained with this chamber in synchrotron $\mathrm{x}$-ray scattering studies of the structure of xenon films adsorbed on the $\operatorname{Ag}(111)$ surface. We chose the $\mathrm{Xe} / \mathrm{Ag}(111)$ system for a number of reasons. Xenon has the largest $\mathrm{x}$-ray cross section of the rare gases, and had already been used in synchrotron diffraction studies of monolayer melting on the $\operatorname{Ag}(111)$ surface. ${ }^{25}$ Comprehensive LEED studies of rare-gas adsorption by Unguris et al. ${ }^{4}$ established the structure and thermodynamic properties of the Xe monolayer, and, to a lesser extent, the bilayer. Since the corrugation of the adatom-substrate potential is less for $\mathrm{Xe} / \mathrm{Ag}(111)$ than on graphite, and there is a large mismatch between the lattice constants of the $\mathrm{Xe}$ monolayer and the $\mathrm{Ag}(111)$ surface, ${ }^{4}$ registry effects seen for the first Xe layer during multilayer growth on graphite ${ }^{13}$ should be minimized.

We have determined structural parameters of multilayer Xe films not only at quasiequilibrium but also under kinetic growth conditions. In probing the multilayer structural parameters inaccessible to LEED (Ref. 4) and He-atom scattering, ${ }^{9}$ we obtain a more sensitive measure of structural perfection in such films than has heretofore been achieved. A preliminary report of this work was published in Ref. 26.

The rest of this paper is organized as follows. Section II contains a description of the experimental technique. Results and discussion are presented in Sec. III, with experiments conducted at quasiequilibrium presented in Sec. II A and nonequilibrium growth experiments in Sec. II B. Section IV contains a summary and conclusions.

\section{EXPERIMENTAL DESCRIPTION}

\section{A. Ultrahigh-vacuum chamber}

Our ultrahigh-vacuum chamber for synchrotron $\mathrm{x}$-ray scattering from films physisorbed on single-crystal surfaces has been described in detail elsewhere, ${ }^{23,24}$ so that we only give a brief description here. The chamber mounts on a standard Huber Model 5020 diffractometer with horizontal $\omega$ and $2 \theta$ axes. It is small enough to allow the entire chamber to be rotated rather than rotating the sample within the chamber to achieve the desired scattering geometry.

Despite its small size, the chamber has several features which make it particularly well suited for investigations of physisorbed and other weakly bound films. The compact layout allows in situ characterization of both the film and single-crystal substrate by LEED and Auger spectroscopy without moving the sample from the $\mathrm{x}$-ray scattering position and without moving the LEED/Auger optics. A commercially available pulse-counting, position-sensitive electron detector system (Quantar Technology) requires a very low incident electron-beam current $(\sim 10 \mathrm{pA})$, thereby minimizing desorption of weakly bound films. Due to the sensitivity of the electron multiplier in front of the LEED detector to photoelectrons produced by the synchrotron x-ray beam, the 
LEED system cannot be used while the x-ray beam is on. However, the LEED and x-ray measurements can be done alternately with sufficient speed that the LEED system provides a convenient means of characterizing the substrate and film just prior to and during the x-ray experiment. The LEED/Auger system was also of great value in performing a "dress rehearsal" of the x-ray experiment prior to bringing the chamber to the synchrotron.

Another feature of our chamber essential to these experiments is a low-temperature capability. Using a commercially available closed-cycle He refrigerator (APD Cryogenics), sample temperatures down to $30 \mathrm{~K}$ could be achieved. In addition, an electron bombardment heater allowed heating to high temperatures (up to $1300 \mathrm{~K}$ ) for cleaning and annealing the Ag substrate.

\section{B. Sample preparation}

The $\operatorname{Ag}(111)$ sample $\left(10 \mathrm{~mm}\right.$ in diameter, miscut $\left.<0.2^{\circ}\right)$ was cleaned by repeated cycles of Xe sputtering ( $8 \mathrm{~h}$ at 350 $\mathrm{K}, 500 \mathrm{eV}$, and $0.25 \mu \mathrm{A} / \mathrm{cm}^{2}$ ) and annealing (30 min, $850 \mathrm{~K}$ ) until there were no detectable impurities on the surface as determined by Auger spectral analysis. ${ }^{23,24}$ After this treatment, the out-of-plane mosaic spread was $\sim 0.3^{\circ}$, and the in-plane mosaic spread was about $0.17^{\circ}$ as measured in X-ray scans transverse to the $\operatorname{Ag}(10)$ crystal truncation rods. The sample temperature was measured by a Au-chromel thermocouple (accuracy $\pm 2 \mathrm{~K}$, relative precision $\pm 0.25 \mathrm{~K}$ ). With a chamber base pressure of $5 \times 10^{-10}$ Torr, the sample remained clean for about eight hours. Xenon films were prepared by exposing the $\operatorname{Ag}(111)$ surface to a constant flux of Xe gas (99.9995\% minimum purity) from a dosing tube situated $1 \mathrm{~cm}$ in front of the substrate.

\section{X-ray scattering}

Our experiments were performed at beam line X18A at the National Synchrotron Light Source. A bent, cylindrical, platinum-coated mirror was used to focus the x-ray beam vertically and horizontally. The sample was aligned using three bulk Bragg reflections of the Ag substrate, $(0 \overline{2} 4)$, (002), and (113). The alignment was then checked by scanning through the $\operatorname{Ag}(10)$ truncation rod at different values of the wave vector transfer perpendicular to the surface, $Q_{\perp}$.

For measurements of the lattice constant of the Xe film, we operated the Huber diffractometer in the four-circle mode. The incident beam was defined by motorized slits with a 3-mm separation horizontally and a $1.5-\mathrm{mm}$ separation vertically. Its wavelength of $1.2208 \AA$ was selected by a double (flat) $\mathrm{Si}(111)$ monochromator. A Ge(111) analyzer was used in the scattered beam to improve resolution in the wave-vector transfer $Q$. In this mode, the spectrometer had a longitudinal, transverse, and out-of-plane resolution width [full width at half maximum (FWHM)] of $1.0 \times 10^{-3}, 1.7$ $\times 10^{-4}$, and $8.3 \times 10^{-2} \AA^{-1}$, respectively, at the bulk Xe anti-Bragg position (see below).

The bulk Ag lattice constant was measured at room temperature and $33 \mathrm{~K}$ with the $\mathrm{Ge}$ analyzer in the scattered beam. Our measured value differs from the reported value ${ }^{27}$ by $\sim 0.02 \%(0.0008 \AA)$. From the radial width of the $\operatorname{Ag}(10)$ truncation rod, we estimate a lateral coherence length $\geqslant 850$ $\AA$ for the bare $\operatorname{Ag}(111)$ surface.

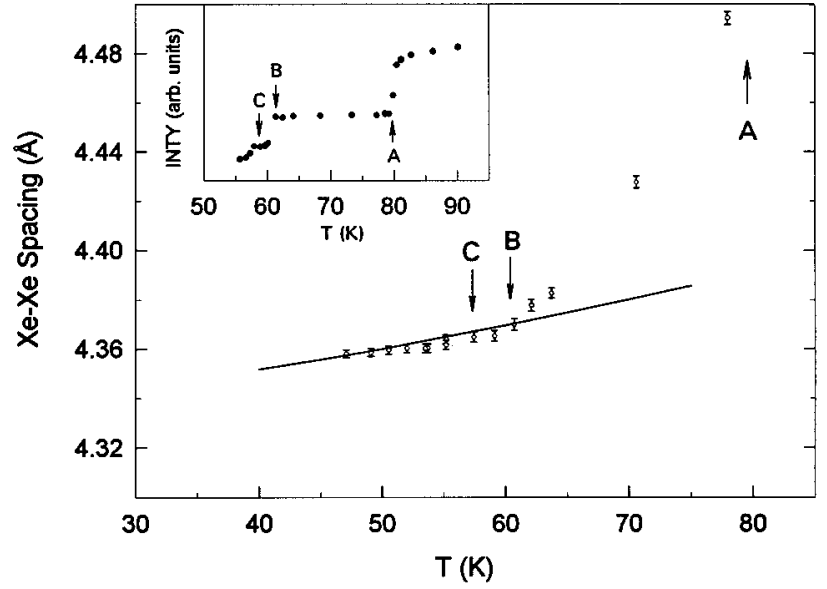

FIG. 1. The Xe-Xe spacing as a function of temperature for a Xe film in equilibrium with a constant Xe flux. The background pressure in the chamber was maintained at $\sim 1.7 \times 10^{-7}$ Torr. The inset shows the $\operatorname{Ag}(10)$ LEED spot intensity observed under the same conditions. In both plots, arrows mark condensation of the first layer $(A)$, the second layer $(B)$, and the bulk $(C)$.

For specular reflectivity measurements, we modified the four-circle diffractometer by mounting a linear drive with a rotary motion on the detector arm so that the detector could be scanned perpendicular to the vertical scattering plane. This so-called "rotating detector five-circle diffractometer'" (Refs. 28 and 29) allowed specular reflection of the x-ray beam from the sample within our UHV chamber. The scattered beam divergence is determined by a pair of slits mounted on a flight path situated in front of the detector. For specular reflectivity measurements, the incident beam wavelength was reduced to $1.0 \AA$ in order to increase the maximum wave-vector transfer perpendicular to the $\operatorname{Ag}(111)$ surface $\left(Q_{\perp}\right)$. The slit sizes were adjusted in this operating mode so that the spectrometer had a longitudinal, transverse, and out-of-plane resolution width (FWHM) of $5.6 \times 10^{-2}$, $3.9 \times 10^{-3}$, and $3.8 \times 10^{-2} \AA^{-1}$, respectively, at the bulk $\mathrm{Xe}\left(00 \frac{3}{2}\right)$ or "anti-Bragg", position.

\section{RESULTS AND DISCUSSION}

\section{A. Quasiequilibrium experiments}

In this section, measurements are reported on films grown under quasiequilibrium conditions by the "isobar" method used in the LEED experiments of Unguris et al. ${ }^{4}$ The lattice constant within a layer was measured with high precision as a function of film thickness by in-plane diffraction scans. Also, nonspecular scans along the $\mathrm{Xe}(01 l)$ rod were used to investigate the layer spacing, stacking sequence, and ultimate thickness of these films.

\section{In-plane diffraction studies}

The Xe films were prepared with the UHV chamber mounted on the $\mathrm{x}$-ray diffractometer. Typically, a background $\mathrm{Xe}$ pressure of $\sim 1.7 \times 10^{-7}$ Torr would be maintained in the chamber as the substrate was cooled slowly from a temperature of $\sim 85 \mathrm{~K}$. The intensity of the $\operatorname{Ag}(10)$ LEED spot intensity recorded for one such run is shown in the inset to Fig. 1. ${ }^{23,24}$ Upon lowering the temperature, three 
sharp drops in the LEED intensity are observed corresponding to monolayer, bilayer, and bulk condensation (labeled $A$, $B$, and $C$, respectively). Use of the in situ LEED system for this "isobar' measurement ${ }^{4,30}$ allowed a precise determination of monolayer and bilayer Xe coverage without resort to a gas ballast. ${ }^{13}$ However, as was the case in the original experiments of this type, ${ }^{4}$ there is insufficient resolution in the temperature and LEED intensity measurements to observe individual layer condensations above two layers.

For the X-ray measurements of the Xe-Xe spacing in the film, the LEED system was turned off after observing the sharp drop in the $\mathrm{Ag}(10)$ spot intensity corresponding to monolayer condensation (see the arrow labeled $A$ in the inset of Fig. 1). As noted above, this prevented severe saturation of the LEED detector. In-plane $\mathrm{x}$-ray scans through the $\mathrm{Xe}(10)$ diffraction rod were then performed using the $\mathrm{Ge}(111)$ analyzer in the scattered beam, as noted in Sec. II. To describe these radial and transverse scans, we use the components of the wave-vector transfer $\mathbf{Q}=\mathbf{k}_{f}-\mathbf{k}_{i}$ parallel and perpendicular to the $\operatorname{Ag}(111)$ surface, $Q_{\|}$and $Q_{\perp}$, respectively. A radial scan is one in which the projection of $\mathbf{Q}$ onto the $\operatorname{Ag}(111)$ surface is directed along the azimuth of the $\mathrm{Xe}(10) \mathrm{rod}$, and $Q_{\|}$is incremented; while, in a transverse scan, $Q_{\|}$is fixed at the distance of the $\mathrm{Xe}(10)$ rod from the origin and the azimuthal angle of $\mathbf{Q}$ is incremented [see the inset on Fig. 6(a) in Ref. 23]. For both types of scan, $Q_{\perp}$ remained constant throughout with a value of $Q_{\perp}$ $=0.2 \AA^{-1}$. As found in earlier work, ${ }^{25}$ both the radial and transverse scans through the $\mathrm{Xe}(10)$ rod can be fit well with a Lorentzian-squared line shape as illustrated in Fig. 2 for scans on multilayer Xe films.

At monolayer coverage, the radial and transverse $\mathrm{x}$-ray scans through the $\mathrm{Xe}(10)$ diffraction rod confirm the results of previous LEED (Ref. 4) and $x-$ ray $^{25}$ experiments of a hexagonal lattice incommensurate but aligned with the substrate. From the radial width of the $\mathrm{Xe}(10)$ and $\mathrm{Ag}(10)$ rods, we estimate the Xe monolayer coherence length to be $\sim 330$ $\AA$ (Ref. 31) compared to $\sim 850 \AA$ for the bare $\operatorname{Ag}(111)$ substrate. The in-plane azimuthal width of the $\mathrm{Xe}(10)$ rod was $2.5^{\circ}-6^{\circ}$, in reasonable agreement with previous measurements. 25

Figure 1 shows the temperature dependence of the $\mathrm{Xe}-\mathrm{Xe}$ spacing in a film obtained from a series of radial scans. These were taken while decreasing the temperature under the same conditions as for the earlier LEED isobar measurement shown in the inset. It is one of several such data sets taken with similar results. In some cases, the LEED system was turned on again briefly at a temperature just above point $B$. In this way, bilayer condensation could be confirmed by observing the second downward step in the Xe(10) LEED spot intensity.

The solid line in Fig. 1 is the Xe-Xe spacing measured by $\mathrm{X}$-ray diffraction for bulk Xe. ${ }^{32}$ We see that the bulk Xe-Xe spacing is reached by monolayer completion (labeled $B$ ) to within our accuracy of $\pm 0.004 \AA{ }^{29,33}$ No deviation from a Lorentzian-squared line shape in the radial scans was observed as the film thickened. In particular, there was no evidence of a splitting of the $\mathrm{Xe}(10)$ peak indicative of a different lattice constant in successive layers of the film as suggested by some computer simulations. ${ }^{34}$
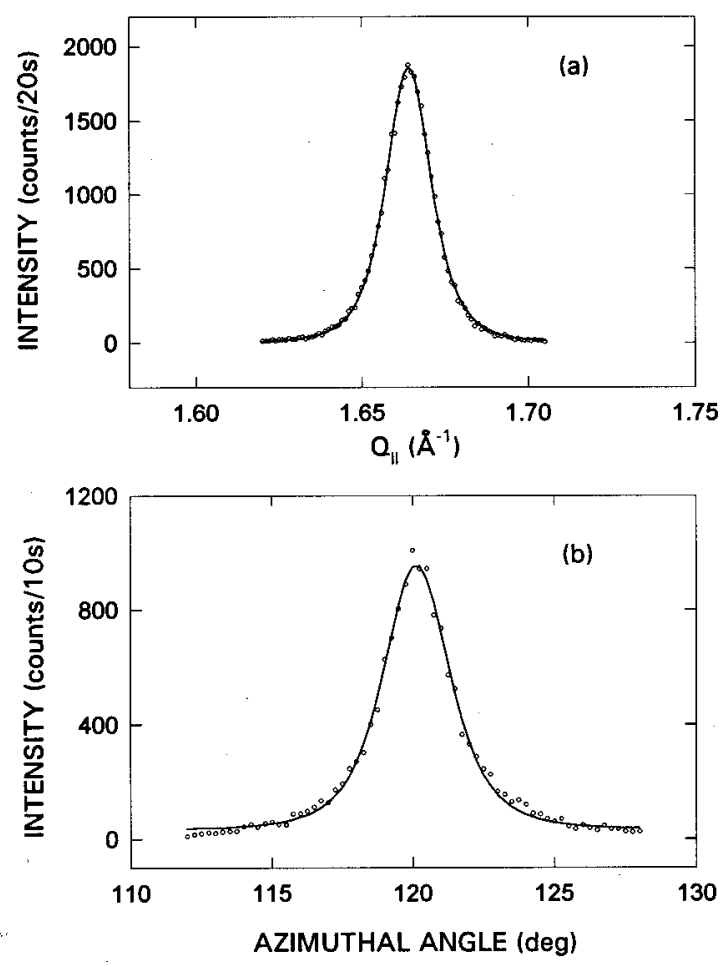

FIG. 2. Typical scans from Xe multilayers on a $\operatorname{Ag}(111)$ substrate. The x-ray wavelength is $1.22 \AA$, and the (constant) perpendicular component of the wave-vector transfer is $Q_{\perp}=0.2 \AA^{-1}$. The scan is taken with a $\mathrm{Ge}(111)$ analyzer in the scattered beam. The solid curves through the data are best fits to a Lorentziansquared line shape as discussed in the text. (a) Radial scan on a film of thickness $\sim 42$ layers prepared at a background pressure of 1.7 $\times 10^{-7}$ Torr and a temperature of $50.5 \mathrm{~K}$. The peak at the $\mathrm{Xe}(10)$ position corresponds to a wave-vector transfer parallel to the surface of $Q_{\|}=1.66 \AA^{-1}$, and it is at the same azimuthal angle $\left(120^{\circ}\right)$ as the $\operatorname{Ag}(10)$ truncation rod. (b) Transverse (azimuthal) scan on a film of thickness $\sim 11$ layers prepared at a background pressure of $5.8 \times 10^{-8}$ Torr and a temperature of $\sim 43 \mathrm{~K}$.

To place a bound on the difference between monolayer and bilayer lattice constants at coexistence, we calculated the line shape to be expected from two independently scattering layers with lattice constants differing by $\Delta a$. Assuming the peaks from each layer to have a Lorentzian-squared line shape, we determined the value of $\Delta a$ at which the scattering summed from both layers would deviate markedly from this line shape. This yielded an estimate of $\Delta a \leqslant 0.005 \AA$ at monolayer-bilayer coexistence.

\section{Nonspecular rod scans}

To investigate the stacking sequence and ultimate thickness of films grown under quasiequilibrium conditions, outof-plane scans were performed along the $\mathrm{Xe}(01 l)$ rod after completion of the in-plane scans. Unlike the specular reflectivity measurements to be discussed below, these nonspecular scans could be performed with the diffractometer in the four-circle mode but, to increase intensity, without the $\mathrm{Ge}$ analyzer in the scattered beam.

The nonspecular rod scan of a multilayer film at a temperature of $47 \mathrm{~K}$ is shown in Fig. 3(a). It shows two peaks 


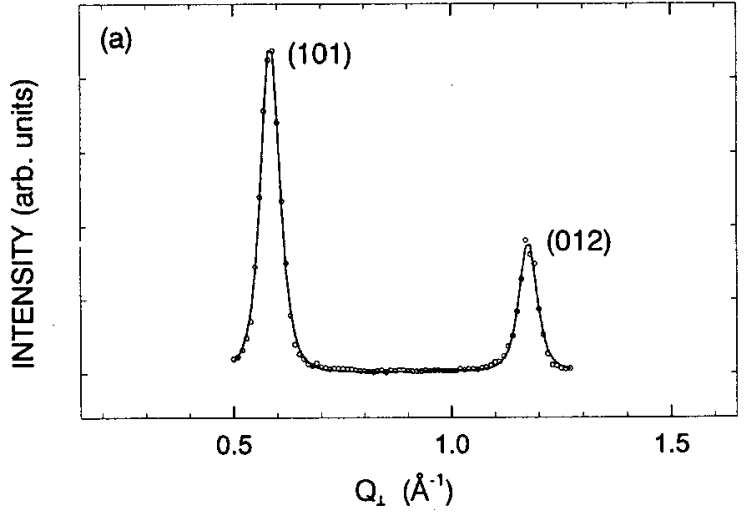

(b)

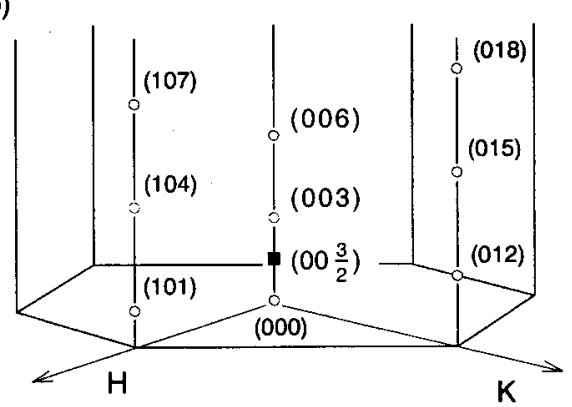

FIG. 3. (a) Scan along the $\mathrm{Xe}(01 l)$ rod for a Xe film of thickness $\geqslant 36$ layers at a temperature of $37 \mathrm{~K}$ and a background pressure of $1.7 \times 10^{-7}$ Torr. The peaks labeled (101) and (012) come from different domains of the film rotated from each other by $60^{\circ}$ about the surface normal. The solid curve represents a Lorentziansquared fit to each peak as described in the text. (b) Schematic representation of reciprocal space relative to the (111) plane of a fcc crystal. The positions of bulk reciprocal-lattice points are indicated by circles and labeled in hexagonal notation. The position labeled $\left(00 \frac{3}{2}\right)$ is an anti-Bragg point of the reciprocal lattice.

one at $Q_{\perp}=0.59 \AA^{-1}$ and the other at twice this value or $Q_{\perp}=1.18 \AA^{-1}$. The first one at $0.59 \AA^{-1}$ corresponds to $2 \pi /\left(3 d_{\mathrm{Xe}}\right)$, where $d_{\mathrm{Xe}}=3.54 \AA$ is the distance between adjacent (111) planes of bulk fcc Xe at this temperature. With the aid of the reciprocal lattice drawn in Fig. 3(b) and labeled in hexagonal notation, ${ }^{35}$ the two peaks in the scan can be indexed as the (101) and (012) reflections of a bulk fcc crystal. They apparently arise from two unequally populated domains of $A B C$ stacking which are rotated $60^{\circ}$ with respect to each other about the surface normal. An $A B A$ stacking of the Xe layers would result in a peak at $Q_{\perp}=0.89 \AA^{-1}$, which is clearly not observed.

The peaks in the nonspecular scan of Fig. 3(a) can be fit well with the same Lorentzian-squared line shape used for fitting the in-plane peaks. ${ }^{23,25}$ From their FWHM of 0.048 $\AA^{-1}$, we estimate a film thickness of $\sim 37$ layers. The thickest film which we have been able to grow under these conditions had a FWHM of $0.028 \AA^{-1}$ corresponding to $\sim 63$ layers. Note that these film thickness estimates are lower bounds, since instrumental resolution has not been deconvoluted from the observed peak width.

\section{B. Nonequilibrium growth experiments}

In this section, we report investigations of the nonequilibrium growth of Xe films. These include observations of tem- poral oscillations in the specularly reflected x-ray intensity analogous to those routinely observed in growth studies of metals and semiconductors by reflection high-energy electron-diffraction (RHEED). Next, specular reflectivity scans on films of nominal thickness of 1-10 layers prepared under various conditions of temperature and pressure are analyzed to determine the spacings and occupancies of the layers. Finally, nonspecular scans on some of these films are analyzed for the film stacking sequence, layer spacings, and layer densities.

\section{Oscillation experiments}

An advantage of x-ray scattering over RHEED is that the scattering can be treated kinematically to a good approximation. To analyze the specular reflectivity of the $\mathrm{Xe} / \mathrm{Ag}(111)$ system, we introduce a simple one-dimensional model describing the Ag substrate and Xe film. The film is assumed to consist of $N$ complete layers of atoms above which are up to three partial layers described by a layer fraction $\theta_{i}\left(\theta_{i} \leqslant 1\right.$, $\theta_{i} \geqslant \theta_{i+1} ; i=1,2$, and 3$)$. Describing the substrate contribution to the intensity by a crystal truncation $\operatorname{rod}^{36,37}$ and ignoring constant prefactors, the x-ray specular reflectivity for a film with three partial layers can be written ${ }^{16,38}$

$$
\begin{aligned}
I= & \mid \frac{f_{\mathrm{Xe}}}{a_{\mathrm{Xe}}^{2}}\left[\frac{1-e^{i N Q_{\perp} d_{\mathrm{Xe}}}}{1-e^{i Q_{\perp} d_{\mathrm{Xe}}}} e^{i Q_{\perp} d_{\mathrm{AgXe}}}\right. \\
& \left.+\sum_{j=1}^{3} \theta_{j} e^{i Q_{\perp}\left(d_{\mathrm{AgXe}}+(N+j-1) d_{\mathrm{Xe}}\right)}\right] \\
& +\left.\frac{f_{\mathrm{Ag}}}{a_{\mathrm{Ag}}^{2}\left(1-e^{\left.-i Q_{\perp} d_{\mathrm{Ag}}\right)}\right.}\right|^{2} \delta\left(Q_{\|}\right),
\end{aligned}
$$

where $f$ and $a$ represent the $Q$-dependent atomic form factor and lattice constant, respectively, for either $\mathrm{Xe}$ or $\mathrm{Ag}$, as denoted by the subscript. The spacings between adjacent film and substrate layers are $d_{\mathrm{Xe}}$ and $d_{\mathrm{Ag}}$, respectively, and $d_{\mathrm{AgXe}}$ is the spacing between the first Xe layer and the Ag substrate. The first term in square brackets in Eq. (1) represents the contribution of the Xe film. The second term is that of the semi-infinite Ag substrate or so-called crystal truncation rod, ${ }^{36}$ which, in the absence of the film, would give rise to a series of bulk Bragg peaks along the $(00 l)$ rod at $l$ $=3,6, \ldots$. These peaks are connected by long tails which fall off as $\sim q_{\perp}^{-2}$, where $q_{\perp}$ measures the distance from the $(00 l)$ Bragg peaks along the specular rod. A Debye-Waller factor has been neglected in Eq. (1), since we will mostly be making comparison with specular scans at low temperature.

In Fig. 4 we show the intensity along the specular $(00 l)$ rod calculated from Eq. (1) for the bare Ag substrate (solid curve), for the substrate with a complete Xe monolayer adsorbed (dashed curve), and for the substrate with a complete Xe bilayer adsorbed (dotted curve). In each of these calculations $\theta_{i}=0(i=1,2$, and 3$)$ as there are no partial Xe layers present. Bulk values of $a_{\mathrm{Ag}}, a_{\mathrm{Xe}}, d_{\mathrm{Ag}}$, and $d_{\mathrm{Xe}}$ have been used for illustrative purposes, since, as shown in Sec. III A, the $\mathrm{Xe}$ film structural parameters reach these values by monolayer completion. ${ }^{39}$ The value of $d_{\mathrm{AgXe}}$ is taken equal to 


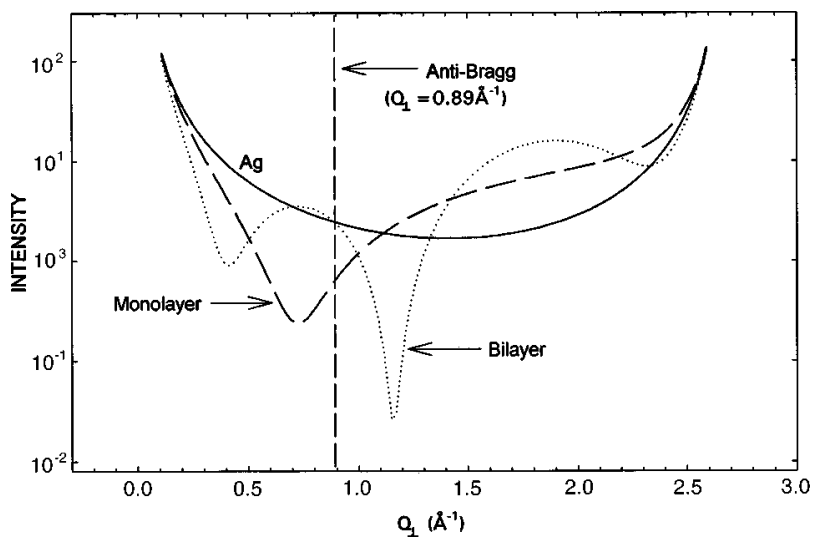

FIG. 4. Intensity along the $(00 l)$ specular rod calculated from Eq. (1) for the bare $\operatorname{Ag}(111)$ substrate (solid curve), a monolayer of Xe adsorbed on this substrate (long-dashed curve), and a Xe bilayer layer adsorbed on the same substrate (dotted curve). Bulk structural parameters are assumed for the Xe film and Ag substrate: $a_{\mathrm{Ag}}$ $=4.07 \AA, a_{\mathrm{Xe}}=4.36 \AA, d_{\mathrm{Ag}}=2.35 \AA$, and $d_{\mathrm{Xe}}=3.54 \AA$. It is assumed that the spacing $d_{\mathrm{AgXe}}$ between the Ag substrate and first Xe layer is equal to $d_{\mathrm{Xe}}$, the layer spacing in the Xe film. The vertical dashed line marks the Xe "anti-Bragg", position indexed $\left(00 \frac{3}{2}\right)$ in hexagonal notation [see Fig. 3(b)].

$d_{\mathrm{Xe}}=3.54 \AA$ consistent with our specular reflectivity data as discussed below and does not represent a simplification of our model.

As can be seen in Fig. 4, the specular reflectivity profile varies dramatically with the addition of each layer of the strongly scattering Xe film. The adsorbed monolayer profile shows destructive interference between the scattering from the $\mathrm{Ag}$ substrate and the Xe overlayer at low values of $Q_{\perp}$, with the intensity reaching a minimum at $Q_{\perp}=0.73 \AA^{-1}$ and then constructive interference with the intensity crossing that of the bare Ag reflectivity at $Q_{\perp}=1.11 \AA^{-1}$. In the case of the adsorbed bilayer, the specular reflectivity curve crosses that of the bare $\mathrm{Ag}$ at four points in the $Q_{\perp}$ range plotted. The crossing at $Q_{\perp}=0.89 \AA^{-1}$ corresponds to an "antiBragg" point of bulk Xe, the location along the specular rod where the two Xe layers scatter $180^{\circ}$ out of phase.

In the model represented by Eq. (1), the specular reflectivity of all films with an odd number of layers will cross each other at the "anti-Bragg" point, and all films with an even number of layers will cross the bare $\mathrm{Ag}$ reflectivity curve at this point. Thus one can begin to see from Fig. 4 that the reflected intensity at the "anti-Bragg'" point should be a periodic function of the Xe coverage with a period of two layers. ${ }^{40}$ This is to be distinguished from the case of homoepitaxial growth where the specular intensity at the "antiBragg" point oscillates with a period of one layer as the coverage is increased. As the film grows thicker and itself approaches a semi-infinite slab, there will be a crossover to a one-layer oscillation period in this model.

At the Xe anti-Bragg point of the specular rod, Eq. (1) predicts the intensity to have a parabolic dependence on coverage with the anticipated periodicity of two layers as illustrated in Fig. 5. This can be seen by setting $N=\theta_{2}=\theta_{3}=0$ in Eq. (1) and calculating the intensity in the range $0 \leqslant \theta_{1}<1$. The curves are continued by incrementing $N$ by 1 and repeating the calculation so that they correspond to ideal layer-by-

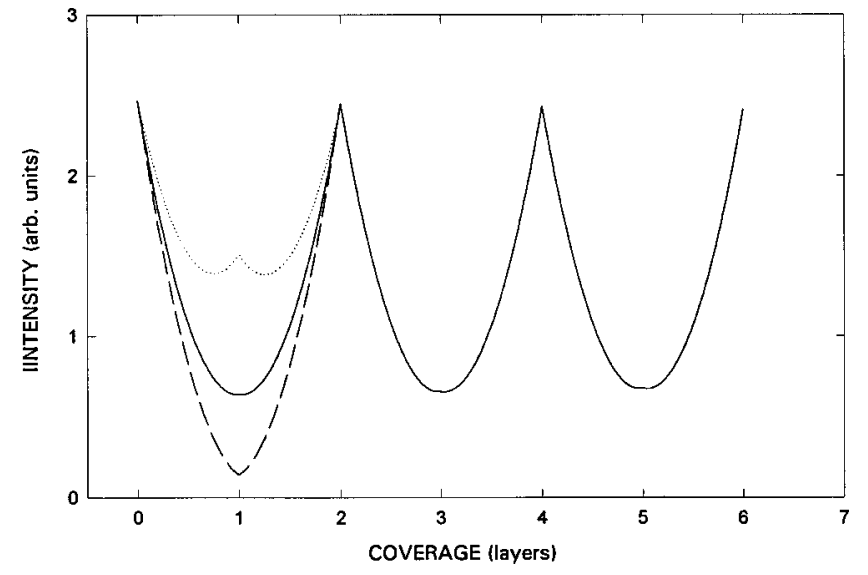

FIG. 5. Calculated X-ray intensity at the $\mathrm{Xe}\left(00 \frac{3}{2}\right)$ or "antiBragg" position $\left(Q_{\perp}=0.89 \AA^{-1}\right)$ as a function of coverage (solid curve). Ideal layer-by-layer growth is assumed; i.e., growth of a layer completes before the next layer begins. As in Fig. $4, d_{\mathrm{AgXe}}$ $=d_{\mathrm{Xe}}=3.54 \AA$. Near the minima the intensity is very sensitive to $d_{\mathrm{AgXe}}$ : the dotted curve corresponds to $d_{\mathrm{AgXe}}=1.1 \times d_{\mathrm{Xe}}$, and the dashed curve to $d_{\mathrm{AgXe}}=0.9 \times d_{\mathrm{Xe}}$.

layer growth (a layer completes before the next layer begins). Notice that the intensity at the oscillation minima does not vanish and is very sensitive to the value of the $\mathrm{Ag}$-Xe spacing, $d_{\mathrm{AgXe}}$, which is varied by $\pm 10 \%$ in Fig. 5 .

It is of interest to compare the calculated curves in Fig. 5 with measurements of the the intensity at the Xe anti-Bragg point of the specular rod during film growth. To achieve the necessary control of the film growth rate, we could not conduct these experiments at the higher pressures required for quasiequilibrium as described in Sec. III A 1. Instead, we cooled the bare $\mathrm{Ag}$ substrate to a temperature at which the sticking probability of the Xe was large (assumed to be close to 1), and exposed the $\operatorname{Ag}(111)$ surface to a constant Xe flux from the dosing tube. Maintaining a background Xe pressure of $5.9 \times 10^{-8}$ Torr in the UHV chamber, resulted in a monolayer deposition time of approximately $10 \mathrm{~min}$ as could be confirmed by LEED experiments. ${ }^{29}$ We shall refer to growth under these conditions as being "kinetic" rather than "isobaric."

The specular intensity at the anti-Bragg point as a function of time is shown in Fig. 6 for kinetic growth at three different substrate temperatures. In each case, damped oscillations having a two-layer period were observed. At $46 \mathrm{~K}$ [Fig. 6(a)] and $43 \mathrm{~K}$ [Fig. 6(b)], we observed four complete oscillations corresponding to the growth of the first eight $\mathrm{Xe}$ layers. The oscillation period is constant to $\leqslant 10 \%$ of a period. Although routinely observed in semiconductor and metal film growth, such oscillations, to our knowledge, have not been previously observed with physisorbed films by any diffraction technique. Multilayer growth of films physisorbed on highly oriented pyrolytic graphite (HOPG) has been studied under quasiequilibrium conditions by ellipsometric techniques; ${ }^{2}$ however, these do not allow one to predict the number of oscillations which might be expected under these kinetic growth conditions.

Similar "oscillation" experiments were performed under a variety of conditions of pressure and temperature. As shown in Fig. 6(c), only one complete oscillation was observed at the lowest attainable temperature of $33 \mathrm{~K}$ at the same pressure of $5.9 \times 10^{-8}$ Torr. Presumably, this is due to 


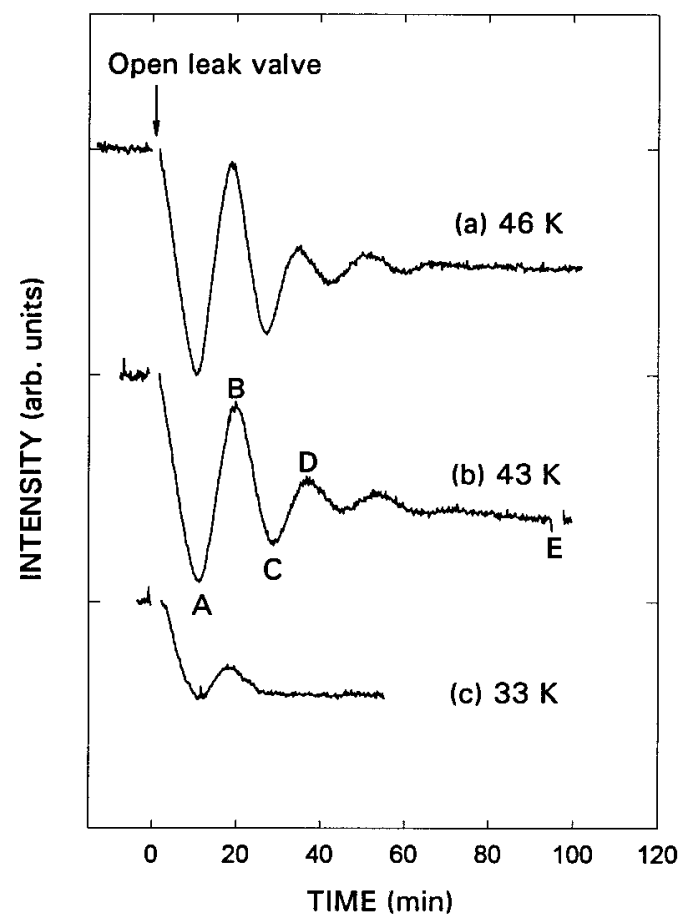

FIG. 6. Time dependence of the x-ray intensity at the $\mathrm{Xe}\left(00 \frac{3}{2}\right)$ "anti-Bragg"' point for various substrate temperatures: (a) $46 \mathrm{~K}$, (b) $43 \mathrm{~K}$, and (c) $33 \mathrm{~K}$. After opening the leak valve, a constant Xe dosing rate is maintained, corresponding to a background pressure in the chamber of $\sim 5.9 \times 10^{-8}$ Torr. In (b), points labeled $A, B, C$, $D$, and $E$ correspond to an approximate Xe film thickness of one, two, three, four, and ten layers, respectively.

a slower Xe diffusion rate at this temperature which results in a growth front spanning more than one layer. We also observed fewer oscillations under growth conditions closer to equilibrium. At a higher temperatures of $55-57 \mathrm{~K}$ and at pressures of both $4.6 \times 10^{-8}$ and $\sim 1.7 \times 10^{-7}$ Torr, oscillations disappeared at film thicknesses of 3-4 layers.

Even under similar conditions of temperature and pressure, we could not consistently reproduce the number of oscillations observed in the specular intensity. One possibility is that the quality of the $\operatorname{Ag}(111)$ surface varied between measurements. In the two cases where the largest number of oscillations were observed [Figs. 6(a) and 6(b)], the substrate was not sputtered prior to annealing. However, we did not attempt a systematic study correlating the number of intensity oscillations observed with sputtering time, annealing time, and the $\operatorname{Ag}(111)$ surface roughness inferred from specular reflectivity scans of the bare substrate.

In no case did the observed specular intensity oscillations have the parabolic shape and constant amplitude of those calculated in Fig. 5. This indicates that the growth is not strictly layer by layer, i.e., more than one partial layer is present above a monolayer coverage. To obtain a more quantitative understanding of the intensity oscillations at the Xe anti-Bragg point of the specular rod, we terminated the film growth at times near the oscillation extrema and performed specular reflectivity scans. These were modeled to determine the density profile of the Xe vacuum interface as described in the next section.

\section{Specular reflectivity scans}

In the first set of specular reflectivity measurements, Xe film growth was investigated under the conditions which gave the largest number of intensity oscillations at the antiBragg point of the specular rod (temperature of $43 \mathrm{~K}$ and background pressure of $\sim 5.9 \times 10^{-8}$ Torr). Dosing of the substrate was terminated at the points labeled $A, B, C, D$, and $E$ in Fig. 6(b), corresponding to approximate Xe film thicknesses of one, two, three, four, and ten layers, respectively. After cooling the sample to $33 \mathrm{~K}$, integrated specular reflectivity scans were taken at these coverages and are shown in Figs. 7 and 8.

As described in Sec. II, the specular reflectivity scans were performed in a horizontal plane with the diffractometer in a five-circle configuration. At each point along the $(00 l)$ rod, the intensity was integrated transverse to the rod by rocking the crystal about a vertical axis. The rocking curves were fitted to a Lorentzian-squared function, ${ }^{23,25}$ assuming a constant background. For comparison with the specular reflectivity calculated from Eq. (1), the area under the Lorentzian-squared fit was corrected for several effects. It was divided by $\cos ^{2} \delta$ where $\delta$ is the scattering angle (as defined in Fig. 2 of Ref. 28) to correct for the x-ray polarization $^{35}$ (the synchrotron radiation is polarized in the horizontal plane). Also, it was multiplied by $\sin ^{2}(\delta / 2) \sim Q_{\perp}^{2}$ to correct for the Lorentz factor, the illuminated sample area, and for the solid angle accepted by the detector. ${ }^{41}$

In Fig. 7, the corrected experimental specular reflectivity scans at nominal monolayer and bilayer coverages (open circles) are compared with fits to the specular intensity calculated from Eq. (1) after folding with the instrumental resolution function. ${ }^{42}$ In all of the fits described below, the values of $d_{\mathrm{Xe}}$ and $d_{\mathrm{Ag}}$ are fixed at their bulk values of 3.54 and 2.35 $\AA$, respectively. At a nominal monolayer coverage [corresponding to terminating the Xe deposition at point $A$ in Fig. 6(b)], one can see that the fit to the specular reflectivity calculated for a complete monolayer $\left(N=1\right.$ and $\theta_{i}=0 ; i=1,2$, and 3) shown by the dashed curve in Fig. 7(a) is poor compared to one in which $N$ and the $\theta_{i}$ are allowed to vary. The best fit (solid curve) is obtained with parameters $N=1, \theta_{1}$ $=0.19$, and $d_{\mathrm{AgXe}}=3.64 \AA .{ }^{43}$

A desorption experiment conducted at the conclusion of the specular scan provides strong support for the latter model. As shown in the inset to Fig. 7(a), the specular intensity at the Xe anti-Bragg point was observed to drop upon heating the sample. This intensity decrement is quantitatively consistent with desorbing a second layer having a fractional occupancy of 0.19 as obtained in the fit to the specular reflectivity. Later, the reflected intensity abruptly rises to that of the bare Ag substrate, signaling the desorption of the first layer. It is noteworthy that desorption experiments of this type cannot be easily performed with more strongly bonded semiconductor and metal films. ${ }^{16-19}$

Similar results are found for a bilayer film grown under the "optimal" conditions for oscillations as in Fig. 6(b) (a temperature of $43 \mathrm{~K}$ and a background pressure in the chamber of $\sim 5.9 \times 10^{-8}$ Torr). Figure 7 (c) contains the specular reflectivity scan on a film grown by terminating Xe deposition at a time corresponding to point $B$ in Fig. 6(b). The dashed curve calculated for two complete layers $(N=2$ and 


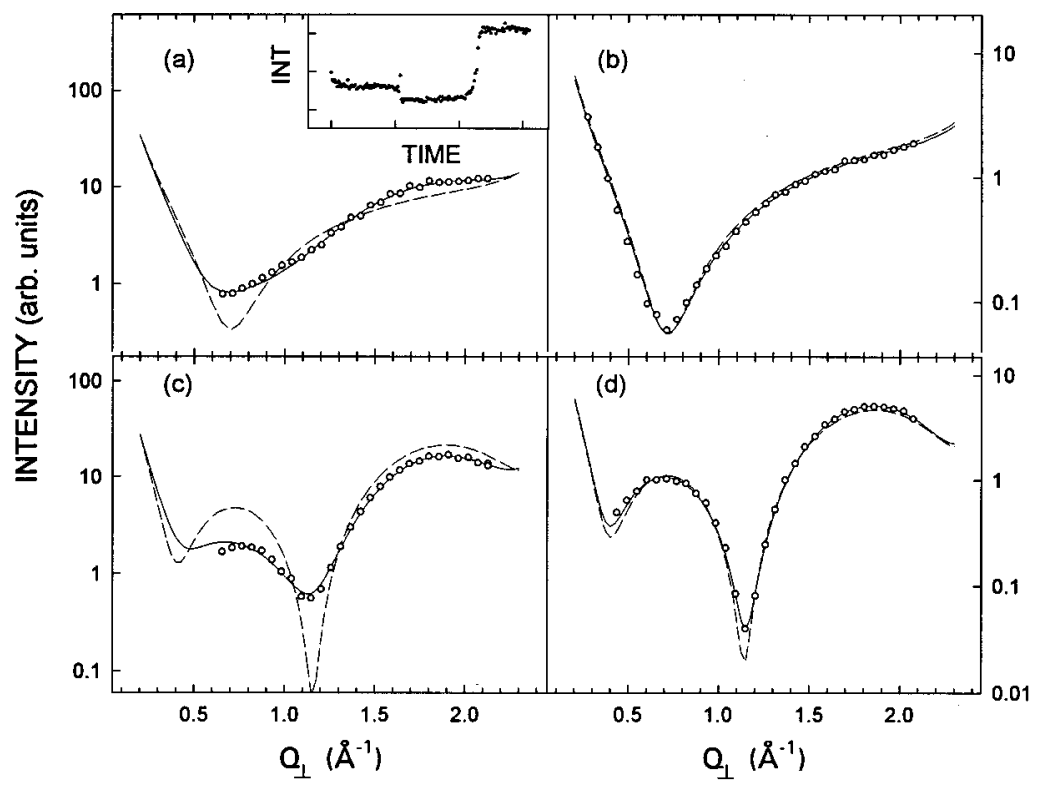

FIG. 7. Specular reflectivity $(00 l)$ scans on monolayer and bilayer films (open circles) taken with an x-ray wavelength of $1.00 \AA$. Scans at $33 \mathrm{~K}$ after growing films under the conditions for which the largest number of intensity oscillations were observed at the anti-Bragg point (temperature of $43 \mathrm{~K}$ and a background pressure of $\sim 5.9 \times 10^{-8} \mathrm{Torr}$ ): (a) nominal monolayer coverage determined by terminating deposition at point $A$ in Fig. 6(b); and (c) nominal bilayer coverage determined by terminating deposition at point $B$ in Fig. 6(b). The inset to (a) shows the intensity at the anti-Bragg point during a desorption experiment on a nominal monolayer film described in the text. There is an abrupt intensity drop after heating for $50 \mathrm{~s}$, and then an abrupt rise at $100 \mathrm{~s}$ to the reflected intensity of the bare Ag surface. Scans below 60 $\mathrm{K}$ on films grown at higher temperatures: (b) Monolayer grown "kinetically", at a temperature of $64 \mathrm{~K}$ and a background pressure of 2.1 $\times 10^{-8}$ Torr. (d) Bilayer grown "isobarically", with a background pressure of $1.7 \times 10^{-7}$ Torr as the sample temperature was reduced from 84 to $61 \mathrm{~K}$. At each point of the specular scan, the intensity has been integrated transverse to the rod, and corrected as described in the text. Note the logarithmic intensity scale. Solid curves are best fits obtained using the partial-layer model of Eq. (1), and dashed lines correspond to film thicknesses of exactly one layer [(a) and (b)] and two layers [(c) and (d)], using the same value of $d_{\mathrm{AgXe}}$ as in the fits. Both calculated and fitted curves have been folded with the instrumental resolution function. Structural parameters obtained from the fits are listed in Table I.

$\theta_{i}=0 ; i=1,2$, and 3) again provides a poor fit compared to one in which $N$ and $\theta_{i}$ are allowed to vary. The best fit (solid curve) is obtained with parameters $N=1, \theta_{1}=0.64, \theta_{2}$ $=0.08, \theta_{3}=0$, and $d_{\mathrm{AgXe}}=3.54 \AA \pm 0.1 \AA$.

To see whether more perfect monolayer and bilayer films could be grown under conditions closer to equilibrium, we made additional specular reflectivity scans at higher temperatures. For example, we grew a film kinetically at a temperature of $64 \mathrm{~K}$ and a background pressure of $2.1 \times 10^{-8}$ Torr. Under these conditions, only a single Xe layer will adsorb on the $\operatorname{Ag}(111)$ surface (see Ref. 4). The specular scan for this film is shown in Fig. 7(b). In this case, we obtained a fit to Eq. (1) with parameters $N=1, \theta_{1}=0.015$, and $\theta_{2}=\theta_{3}=0$. The solid curve corresponding to these parameters ${ }^{44}$ is indistinguishable from the dashed curve in Fig. 7(b) calculated for a complete monolayer.

In another experiment, we grew a bilayer film isobarically, as described in Sec. III A 1, at a background pressure of $1.7 \times 10^{-7}$ Torr, while slowly cooling the Ag substrate from 84 to $61 \mathrm{~K}$. The lower temperature corresponds to the foot of the second-layer substep (just above point $C$ in the inset to Fig. 1). Fitting the specular scan from this film in Fig. 7(d), we obtained a best fit with parameters $N=1, \theta_{1}$ $=0.97, \theta_{2}=0.06$, and $\theta_{3}=0$. In Fig. 7(d), the solid curve generated from these parameters is close to that of the dashed curve corresponding to two complete layers. ${ }^{44}$
The fit to the monolayer specular scan in Fig. 7(b) and and the bilayer scan in Fig. 7(d) gave values of the Ag-Xe distance $d_{\mathrm{AgXe}}=3.58$ and $3.64 \AA$, respectively. These values agree within the experimental uncertainty with the value of $3.55 \AA$ obtained from a dynamical analysis of a LEED experiment. ${ }^{45}$ For both the $\mathrm{x}$-ray and LEED experiments, the uncertainty in $d_{\mathrm{AgXe}}$ is $\pm 0.1 \AA$. Table I summarizes the structural parameters obtained by fitting the monolayer and bilayer specular reflectivity scans in Fig. 7 to Eq. (1).

Above two layers, it was difficult to grow films having as high a degree of structural perfection as those at lower coverage. Films grown kinetically under the same conditions as in Fig. 6(b) (a temperature of $43 \mathrm{~K}$ and a background pressure of $\sim 5.9 \times 10^{-8}$ Torr) with deposition terminated at point $C$ ( $\sim 3$ layers) showed the expected threefold modulation in the specular reflectivity scan as in Fig. 8(a). However, the intensity extrema in the measured reflectivity were much smaller than those calculated from Eq. (1) for exactly three layers (dashed curve). The agreement did not improve significantly by deposition at higher temperatures as in Fig. 8 (b). In this case, a background pressure of $\sim 8.8$ $\times 10^{-8}$ Torr was maintained with Xe deposition beginning at a temperature of $81 \mathrm{~K}$ which was slowly reduced to $59 \mathrm{~K}$ while monitoring the number of intensity oscillations at the anti-Bragg point of the Xe specular rod.

The modulation in the specular scans became even weaker at still higher coverages. The specular reflectivity 


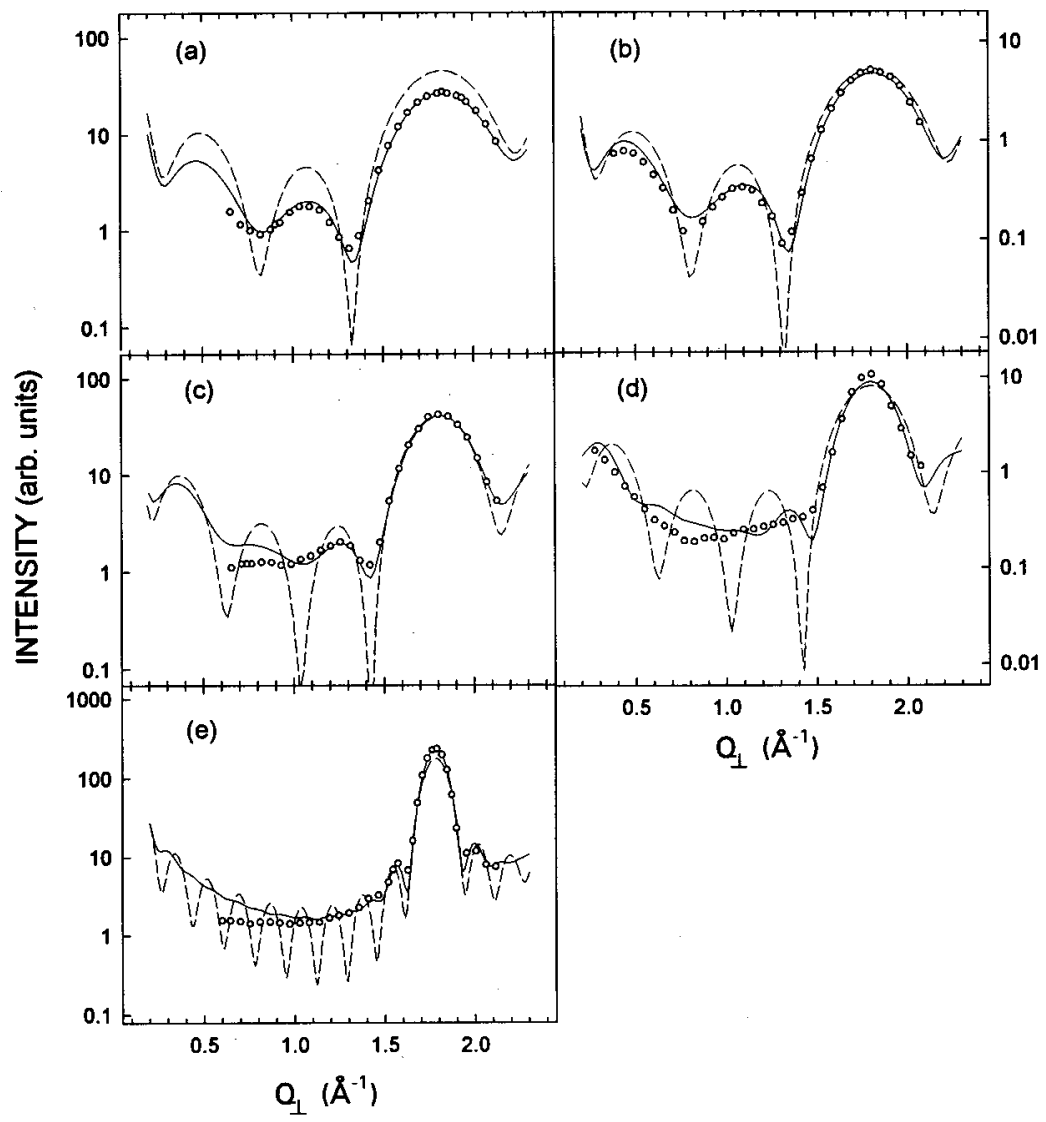

FIG. 8. Integrated specular reflectivity scans of Xe films at nominal coverages of three [(a) and (b)], four [(c) and (d)], and ten [(e)] layers taken with an X-ray wavelength of $1.00 \AA$. Solid curves are best fits to the Gaussian model of Eq. (2), and the corresponding parameters are listed in Table II. Dashed curves are calculated from Eq. (1) for an integer number of layers at the nominal thickness (using the same value of $d_{\mathrm{AgXe}}$ inferred from the Gaussian model). Scans (a), (c), and (e) were taken at a temperature of $33 \mathrm{~K}$ on films grown under the conditions for which the largest number of intensity oscillations were observed at the anti-Bragg point (temperature of $43 \mathrm{~K}$ and a background pressure of $\sim 5.9 \times 10^{-8}$ Torr); deposition was terminated at points $C, D$, and $E$, respectively, in Fig. 6(b). Scans in (b) and (d) were taken on Xe films grown at higher temperatures: (b) Nominal three-layer film prepared by dosing the substrate at a constant rate (background pressure of $8.8 \times 10^{-8}$ Torr) and slowly reducing the substrate temperature from 81 to $59 \mathrm{~K}$ while monitoring the $\mathrm{x}$-ray intensity at the anti-Bragg point of the Xe specular rod. (d) Nominal four-layer film prepared in the same way as (b) with a background pressure of $5.8 \times 10^{-8}$ Torr and growth temperatures in the range $78-56 \mathrm{~K}$.

scans in Figs. 8(c) and 8(e) were obtained by depositing Xe under the optimal conditions for temporal intensity oscillations and terminating at points $D(\sim 4$ layers $)$ and $E(\sim 10$ layers), respectively, in Fig. 6(b). Neither scan shows the expected $n$-fold modulation, with only the principal maximum and one or two secondary maxima being observed. Again, growth at higher temperatures did not improve the situation as can be seen for $\sim 4$-layer coverage in Fig. 8(d), where deposition at a background pressure of $\sim 5.8$ $\times 10^{-8}$ Torr began at a temperature of $78 \mathrm{~K}$ and ended at 56 $\mathrm{K}$.

The weak modulation in these specular scans suggests that these thicker films are rougher at the vacuum interface. To fit the specular reflectivity curves of these films, we have considered a simpler model rather than extending that of Eq. (1) to include more partial layers. The model assumes domains of an integer number of layers $N$ scattering independently. These domains have a Gaussian distribution of thicknesses yielding three adjustable parameters in the model: the mean number of layers in a domain $n$, the standard deviation $\sigma$ of $n$, and the distance $d_{\mathrm{AgXe}}$ between the top layer of the $\operatorname{Ag}(111)$ substrate and the first Xe layer. In this model, the specular intensity is proportional to the expression

$$
\begin{aligned}
& I \propto \sum_{N=1}^{2 n}\left[\frac{1}{\sigma \sqrt{2 \pi}} e^{-(1 / 2)[(n-N) / \sigma]^{2}} \mid \frac{f_{\mathrm{Xe}}\left(1-e^{i N Q_{\perp} d_{\mathrm{Xe}}}\right)}{a_{\mathrm{Xe}}^{2}\left(1-e^{\left.i Q_{\perp} d_{\mathrm{Xe}}\right)}\right.}\right. \\
& \left.\quad \times e^{i Q_{\perp} d_{\mathrm{AgXe}}}+\left.\frac{f_{\mathrm{Ag}}}{a_{\mathrm{Ag}}^{2}\left(1-e^{\left.-i Q_{\perp} d_{\mathrm{Ag}}\right)}\right.}\right|^{2}\right] \delta\left(Q_{\|}\right) .
\end{aligned}
$$

The solid curves in Fig. 8 represent the best fit to the specular reflectivity curves at coverages of three layers and above using the Gaussian model of Eq. (2). Fitting parameters at each coverage are given in Table II. In all cases, the fit is much better than for an integer number of layers at the nominal coverage (dashed curves). Values of the Ag-Xe distance $d_{\mathrm{AgXe}}$ are in the range 3.51-3.59 $\mathrm{\AA}$. 
TABLE I. Results of analyzing specular reflectivity scans of nominal monolayer and bilayer Xe films adsorbed on a $\operatorname{Ag}(111)$ surface. Structural parameters result from best fits to the model of Eq. (1): $\theta_{i}$ is the fractional occupancy of the $i$ th layer above $N$ complete layers, $d_{\mathrm{AgXe}}$ is the distance between the $\operatorname{Ag}(111)$ surface and the first Xe layer, and $T$ is the temperature of the reflectivity scan.

\begin{tabular}{lcccccr}
\hline \hline $\begin{array}{l}\text { Nominal } \\
\text { thickness }\end{array}$ & $N$ & $\theta_{1}$ & $\theta_{2}$ & $\theta_{3}$ & $d_{\text {AgXe }}(\AA)$ & $T(\mathrm{~K})$ \\
\hline 1 & 1 & 0.19 & 0 & 0 & $3.64^{\mathrm{a}}$ & 33 \\
1 & 1 & 0.01 & 0 & 0 & 3.58 & $\sim 60$ \\
2 & 1 & 0.64 & 0.08 & 0 & 3.54 & 33 \\
2 & 1 & 0.97 & 0.06 & 0 & 3.64 & $\sim 60$ \\
\hline \hline
\end{tabular}

${ }^{\mathrm{a}}$ See Ref. 43.

The presence of independently scattering domains in three-layer and thicker films suggested by these specular reflectivity scans is consistent with our interpretation of the nonspecular scans on thicker films. As we have seen in Fig. 3 , a scan along the $\mathrm{Xe}(01 l)$ rod for the much thicker film ( $\geqslant 36$ layers) grown under quasiequilibrium conditions indicated the presence of unequally populated crystalline film domains separated azimuthally by an angle of $60^{\circ}$. Analysis of all specular scans at coverages above three layers indicates that these domains are well described by a Gaussian thickness distribution.

\section{Nonspecular scans}

Although analysis of the specular scans in Sec. III B 2 allows a characterization of the atomic density profile at the $\mathrm{Xe} / \mathrm{vacuum}$ interface, it does not provide any information on the lateral order in the film near this interface. In Sec. III A 2, we reported nonspecular rod scans of relatively thick films grown under quasiequilibrium conditions which showed Bragg peaks along the $\mathrm{Xe}(01 \mathrm{l})$ rod consistent with film domains separated azimuthally by $60^{\circ}$. The peak positions and widths allowed determination of the layer spacing and thickness of the films which had predominantly an $A B C$ stacking sequence.

In this section, we present similar scans for thinner films grown kinetically under various conditions of temperature and pressure. Besides investigating the domain structure of

TABLE II. Results of analyzing specular reflectivity scans of Xe films adsorbed on a $\operatorname{Ag}(111)$ surface with a nominal thickness greater than or equal to three layers. Structural parameters correspond to best fits to the Gaussian model of Eq. (2), where $n$ is the mean number of layers in a domain with standard deviation $\sigma$. $d_{\mathrm{AgXe}}$ is the distance between the $\operatorname{Ag}(111)$ surface and the first $\mathrm{Xe}$ layer, and $T$ is the temperature of the reflectivity scan.

\begin{tabular}{rcccc}
\hline \hline $\begin{array}{c}\text { Nominal } \\
\text { thickness }\end{array}$ & $n$ & $\sigma$ & $d_{\text {AgXe }}(\AA)$ & $T(\mathrm{~K})$ \\
\hline 3 & 2.98 & 0.55 & 3.51 & 33 \\
3 & 3.21 & 0.50 & 3.59 & 57 \\
4 & 3.88 & 0.78 & 3.50 & 33 \\
4 & 4.80 & 0.98 & 3.56 & 55 \\
10 & 10.5 & 1.84 & 3.54 & $\sim 33$ \\
\hline \hline
\end{tabular}

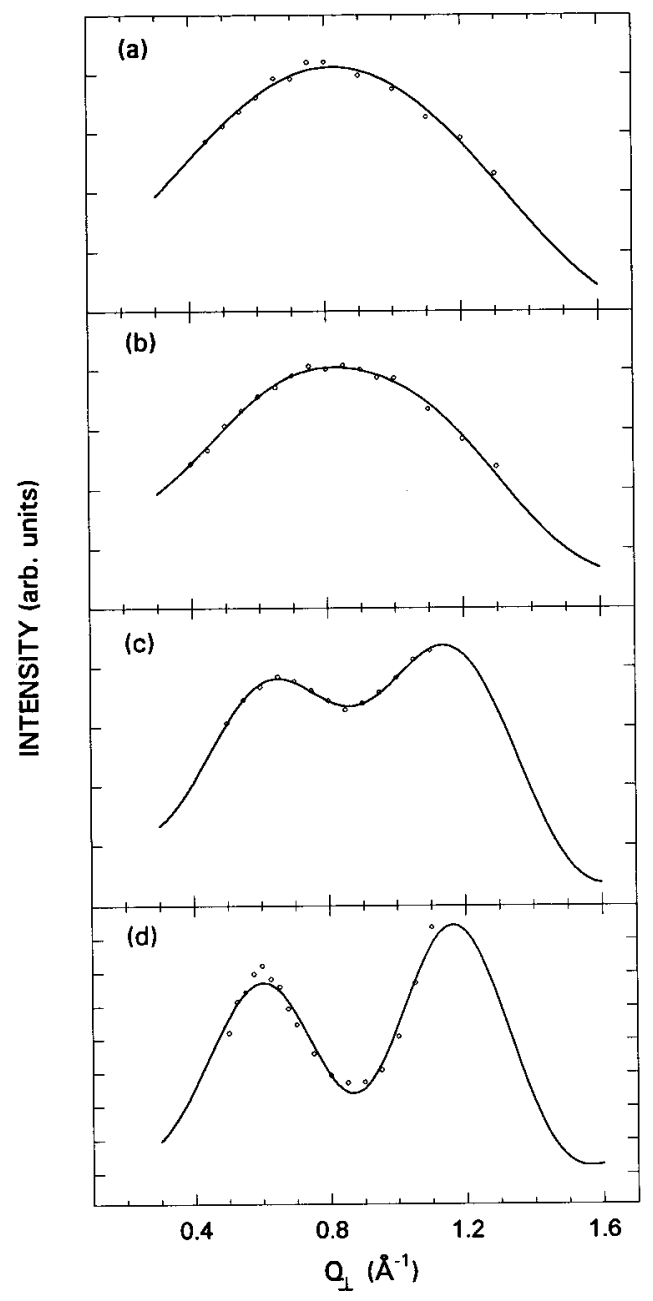

FIG. 9. Integrated nonspecular scans of the $\mathrm{Xe}(01 l)$ rod taken in the four-circle diffractometer mode (incident and exit angles equal) for films of various thicknesses. Solid curves are best fits to the model represented by Eqs. (3)-(5). Corresponding structural parameters are listed in Table III. (a) Bilayer film prepared by the LEED kinetic isotherm method (a substrate temperature of $34 \mathrm{~K}$ and a background pressure of $2.7 \times 10^{-8}$ Torr). Scan performed at $34 \mathrm{~K}$ at a wavelength of $1.22 \AA$. (b) Trilayer film prepared by the LEED kinetic isotherm method (a substrate temperature of $37 \mathrm{~K}$ and a background pressure of $3.2 \times 10^{-8}$ Torr). The scan is performed at $37 \mathrm{~K}$ at a wavelength of $1.22 \AA$. (c) Same nominal trilayer film as in Fig. 8(b). The scan is performed at $57 \mathrm{~K}$ at a wavelength of $1.00 \AA$. (d) Same nominal four-layer film as in Fig. 8(d). The scan is performed at $55 \mathrm{~K}$ at a wavelength of $1.00 \AA$.

the films, our interest is in examining their stacking sequence in more detail. In two cases, we analyze nonspecular scans on the same films for which the specular reflectivity results were presented in Sec. III B 2. These films, having a nominal thickness of three and four layers, can be used to address whether a Xe film initially grows on the $\mathrm{Ag}(111)$ substrate with an $A B C$ stacking sequence of the bulk fcc crystal, or whether it has $A B A$ stacking faults characteristic of a hcp crystal.

Unlike specular reflectivity measurements, these nonspecular scans could be performed while the diffractometer was still in the four-circle mode. Scans along the $\mathrm{Xe}(01 l)$ rod are shown in Fig. 9 for four kinetically grown films which have nominal thicknesses ranging from two to four 
layers. As with the specular scans, the intensity plotted at each value of $Q_{\perp}$ in the nonspecular scans of Fig. 9 represents the integrated intensity along a cut in reciprocal space transverse to the $(01 l)$ rod. These transverse scans were fitted to a Lorentzian-squared function, assuming a constant background. The product of the peak height and the FWHM of the Lorentzian-squared fit is proportional to the integrated intensity.

To analyze the nonspecular scans, we follow closely the approach of Vlieg et al. for the case of homoepitaxial crystal growth. ${ }^{46} \mathrm{We}$ assume that the Xe film is laterally uncorrelated with the $\operatorname{Ag}(111)$ substrate, and introduce a two-level model of the Xe/vacuum interface. That is, we consider a film of $N$ complete layers covered by islands of monolayer thickness which occupy a fraction $\theta$ of the available area, and whose in-plane correlations are described by a Gaussian distribution. ${ }^{46}$ For simplicity, we did not consider partial occupancy of higher layers. The derivation of the expression for the total intensity along the $(01 l)$ rod (Bragg plus diffuse) is given in Ref. 47. Ignoring constant prefactors, we obtain

$$
\begin{aligned}
I= & \frac{A}{A_{u}}\left[\left|E_{C N}\right|^{2}+f_{\mathrm{Xe}}^{2} \theta+\theta E_{C N}^{*} f(N+1) e^{i Q_{\perp} N d_{\mathrm{Xe}}}\right. \\
& \left.+\theta E_{C N} f^{*}(N+1) e^{-i Q_{\perp} N d_{\mathrm{Xe}}}\right]
\end{aligned}
$$

Here $A$ is the sample area participating in diffraction, $A_{u}$ is the area of the Xe unit cell, and $f_{\mathrm{Xe}}$ is the $Q$-dependent $\mathrm{Xe}$ atomic form factor. The "vertical" scattering amplitude $E_{C N}$ from one column of the unit cell consisting of $N$ complete layers is

$$
E_{C N}=\sum_{n_{3}=0}^{N-1} f\left(n_{3}\right) e^{i Q_{\perp} d_{\mathrm{Xe}}}
$$

As in Eqs. (1) and (2), $Q_{\perp}$ is the component of the wavevector transfer perpendicular to the surface, and $d_{\mathrm{Xe}}$ is the distance between adjacent Xe layers. In both Eqs. (3) and (4), the term $f\left(n_{3}\right)$ is the product of $f_{\mathrm{Xe}}$ and a phase factor which depends on the type of the layer $n_{3}$. For " $A$,', ' $B$,', and ' $C$ ' layers of a fcc structure, $f\left(n_{3}\right)$ is, respectively, $f_{\mathrm{Xe}}, f_{\mathrm{Xe}} e^{i Q_{\|}\left[(2 / 3) a_{1}+(1 / 3) a_{2}\right]}$, and $f_{\mathrm{Xe}} e^{i Q_{\|}\left[(1 / 3) a_{1}+(2 / 3) a_{2}\right]}$. Note that the layer argument $N+1$ of $f$ in Eq. (3) refers to the top (partial) layer. Explicit expressions for the $E_{C N}$ for threeand four-layer films with both $A B A$ and $A B C$ stacking are given in Ref. 47.

The nonspecular scans on the three- and four-layer films cannot be fit well by models assuming either perfect $A B C$ or $A B A$ stacking. ${ }^{29}$ For example, fitting the nonspecular scan of the three-layer film in Fig. 9(b) to a model with perfect $A B C$ stacking gives a broad double-peak structure similar to the solid curves in Figs. 9(c) and 9(d). On the other hand, a model of perfect $A B A$ stacking produces a single peak centered at $Q_{\perp}=0.89 \AA^{-1}$ which is too narrow to fit the data.

In order to investigate the effect of stacking faults, we have considered a model in which domains of perfect $A B C$ and $A B A$ stacking scatter incoherently. For each stacking domain, there may be a second domain rotated about the surface normal from the first one by $60^{\circ}$. For such a model, the total intensity along the $(10 l)$ rod can be written as
TABLE III. Results of analyzing scans of the nonspecular $\mathrm{Xe}(10 l)$ rod for films adsorbed on a $\mathrm{Ag}(111)$ surface with a nominal thickness of 2-4 layers. Structural parameters result from best fits to the model of Eq. (3). $\theta$ is the fractional occupancy of the layer above $N$ complete layers, $\alpha$ is the fraction of the film with $A B A$ stacking, $\beta$ is the fraction of the film which is rotated about the surface normal by $60^{\circ}$, and $T$ is the scan temperature.

\begin{tabular}{lccccc}
\hline \hline $\begin{array}{l}\text { Nominal } \\
\text { thickness }\end{array}$ & $N$ & $\theta$ & $\alpha$ & $\beta$ & $T(\mathrm{~K})$ \\
\hline 2 & 2 & 0.04 & 0 & 0.57 & 33 \\
3 & 3 & 0.09 & 0.34 & 0.57 & 33 \\
$3^{\mathrm{a}}$ & 3 & 0.62 & 0.23 & 0.62 & 57 \\
$4^{\mathrm{b}}$ & 4 & 0.49 & 0.11 & 0.63 & 55 \\
\hline \hline
\end{tabular}

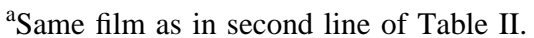

${ }^{\mathrm{b}}$ Same film as in fourth line of Table II.

$$
\begin{aligned}
I= & (1-\alpha)\left[(1-\beta) I_{A B C 1}+\beta I_{A B C 2}\right]+\alpha\left[(1-\gamma) I_{A B A 1}\right. \\
& \left.+\gamma I_{A B A 2}\right],
\end{aligned}
$$

where $I_{A B C 1}$ denotes the intensity contributed from the first domain of $A B C$ stacking, and $I_{A B C 2}$ comes from a second domain of the same stacking rotated $60^{\circ}$ azimuthally from the first. A similar notation applies to the domains having $A B A$ stacking. $\alpha$ is the fraction of domains having $A B A$ stacking, while $\beta$ and $\gamma$ are, respectively, the fraction of the $A B C$ and $A B A$ domains rotated by $60^{\circ}$. Each intensity term in Eq. (5) has the form of Eq. (3). Explicit expressions for these terms for three- and four-layer films with both $A B A$ and $A B C$ stacking are given in Ref. 47.

The nonspecular scans in Fig. 9 were fitted to Eq. (5) after making appropriate corrections for the Lorentz factor, scattering geometry, and instrumental resolution as described in Ref. 48. The best fits are shown by the solid curves, and the fitting parameters are listed in Table III. The parameter $\gamma$ was set to zero because the peak resulting from the $60^{\circ}$ rotated domain of $A B A$ stacking was far out of the scan range.

The films on which the nonspecular scans in Figs. 9(a) and 9(b) were performed were grown kinetically by dosing the substrate at temperatures of $\sim 34$ and $\sim 37 \mathrm{~K}$, respectively, while monitoring the LEED Xe(10) spot intensity. The film thickness was determined by the total deposition time, assuming unity sticking coefficient, and the previously measured monolayer adsorption time. ${ }^{29}$ Since the film in Fig. 9(a) is only two layers thick, the question of stacking sequence does not arise; however, the width of the peak in the (10l) rod scan provides evidence of film domains azimuthally separated by $60^{\circ}$. Fitting the peak width required about an equal population of rotated and nonrotated domains ( $\beta$ $=0.57$ ). For a similarly prepared three-layer film, the fit to the $(10 l)$ rod scan in Fig. 9(b) gave the same azimuthal distribution of domains $(\beta=0.57)$. About a third of the film had $A B A$ stacking $(\alpha=0.34)$, and there was only a small fourth-layer occupancy $(\theta=0.09)$. As noted above, if only $A B C$ stacking were present, there would be a double-peak structure in the calculated profile as in Figs. 9(c) and 9(d). This line shape would persist even if more than one partial layer were included in the model.

We can compare these results with nonspecular scans of three- and four-layer films grown kinetically at higher tem- 


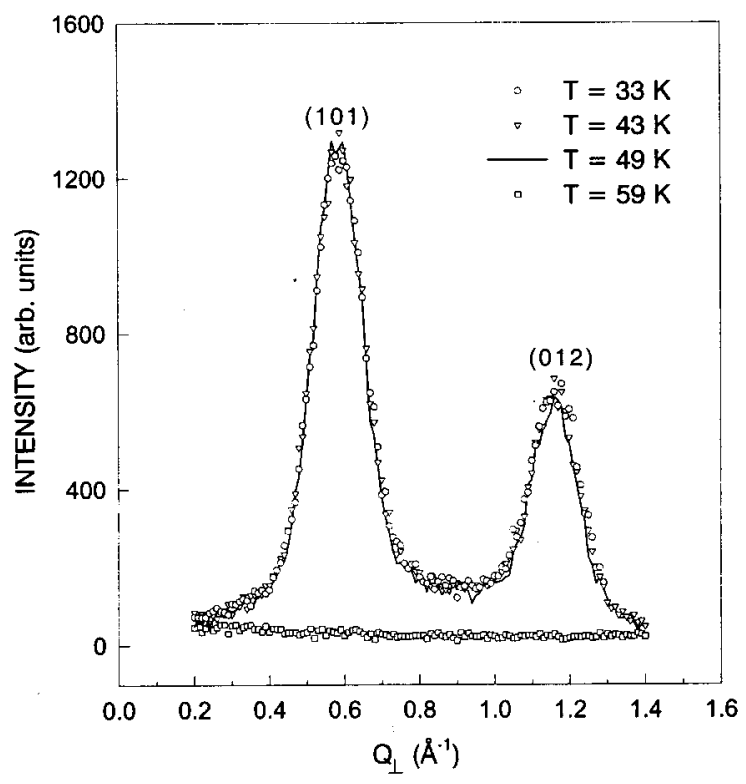

FIG. 10. Temperature dependence of the scans along the $(01 l)$ rod of a Xe film of thickness $\geqslant 10$ layers: (a) $33 \mathrm{~K}$, (b) $43 \mathrm{~K}$, (c) 49 $\mathrm{K}$, and (d) $59 \mathrm{~K}$. Note that multilayer desorption occurs at $59 \mathrm{~K}$, leaving only the monolayer. The film was grown at a temperature of $33 \mathrm{~K}$ with a background pressure in the chamber of 4.0 $\times 10^{-8}$ Torr while monitoring the intensity at the anti-Bragg point of the Xe specular rod. The scans were performed in the four-circle operating mode of the diffractometer at a wavelength of $1.22 \AA$.

perature $(56-81 \mathrm{~K})$ on which specular scans also were performed [Figs. 8(b) and 8(d); second and fourth entries in Table II]. For these films growth was monitored by observing intensity oscillations at the anti-Bragg point of the Xe specular rod. Fits to the $(10 l)$ rod scans of both films [Figs. 9 (c) and $9(\mathrm{~d})$ ] yield a slightly larger fraction of azimuthally rotated domains $(\beta=0.62-0.63)$ (Ref. 49) than for films grown at lower temperature, but smaller fractions of $A B A$ stacking $(\alpha=0.23$ and 0.11 for the three- and four-layer films, respectively). The thickness of both films exceeds its nominal value with the best fit giving a total coverage of 3.62 layers for the three-layer film, and 4.49 for the fourlayer film. We note that analysis of the specular reflectivity of these films using the Gaussian thickness model also gave mean thicknesses greater than the nominal value: $n=3.21$ (three-layer film) and $n=4.80$ (four-layer film). Although the different models of the Xe/vacuum interface used in analyzing the specular and nonspecular scans of these films make comparison of the results difficult, both types of rod scans indicate that the films grown at higher temperature are both thicker and rougher than those grown at the lowest attainable temperatures $\leqslant 37 \mathrm{~K}$.

From analyzing the nonspecular scans in Fig. 9, we see a tendency for the fraction of $A B A$ stacking to decrease in the thicker films, implying that the $A B A$ stacking faults are concentrated near the $\operatorname{Ag}(111)$ surface. This possibility was investigated further by depositing a thicker film (dosing time of $\sim 2 \mathrm{~h}$ ) with the substrate held at a temperature of $33 \mathrm{~K}$ and a background pressure in the chamber of $4.0 \times 10^{-8}$ Torr. The $(01 l)$ rod scans on this film are shown as a function of temperature in Fig. 10. Again, we see a double-peak pattern as in Fig. 3(a) consistent with scattering from two film domains azimuthally separated by $60^{\circ}$. From the FWHM of these peaks $\left(\sim 0.17 \AA^{-1}\right)$, we infer a film thickness of $\geqslant 10$ layers. The nonvanishing intensity observed halfway between the two peaks is indicative of some fraction of the film having $A B A$ stacking. Fitting the scans to the model represented by Eq. (5), ${ }^{50}$ we estimate the fraction of the film with $A B A$ stacking to be $\alpha \approx 0.10$, which is comparable to the fraction found for the four-layer film (see Table III). That the $A B A$ fraction did not decrease further as the film thickness increased from four to ten layers may be due to the low growth temperature $(33 \mathrm{~K})$ of the ten-layer film.

It was of interest to determine whether annealing of the film could reduce the fraction of $A B A$ stacking faults. However, we see no temperature dependence of $(01 l)$ rod scans in Fig. 10 as the film is heated up to the multilayer desorption temperature of $59 \mathrm{~K}$ where only the uniform intensity expected for the monolayer (01) rod remains (square symbols in Fig. 10). We conclude that it is impossible to remove the stacking faults by annealing.

Thus the picture which emerges from these nonspecular scans is that the $A B A$ stacking faults occur predominantly near the $\operatorname{Ag}(111)$ interface during the initial stages of film growth. As will be discussed further below, these stacking faults may be pinned to steps in the Ag surface and hence resist annealing; or the stacking faults may result from strain induced in the first few layers of the film as a result of their locking to the upper Xe layers which have the bulk lattice constant.

\section{SUMMARY AND CONCLUSIONS}

In this paper, we have reported synchrotron x-ray scattering studies of the structure and growth of Xe films adsorbed on a $\operatorname{Ag}(111)$ surface. Our results extend previous experimental studies of this system in several respects. For measurements of intraplanar lattice constants, the use of a synchrotron $x$-ray source provides higher precision than achievable with LEED and elastic He-atom scattering. More significantly, the synchrotron $\mathrm{x}$-ray technique allows us to address issues of "out-of-plane" structure which have not been dealt with quantitatively by these other techniques. Specular and nonspecular scans permit characterization of the atomic density profile at the Xe/vacuum interface and the stacking sequence in these films. We have been able to assess whether layer-by-layer growth occurs in these films above three layers, to detect the presence of stacking faults in the initial stages of film growth, and to determine the ultimate film thickness. Crucial to the success of these experiments has been the development of an ultrahigh-vacuum chamber which allows synchrotron x-ray scattering to be performed on films of quality comparable to those investigated earlier by other techniques, ${ }^{9-11}$ and of better quality than in previous x-ray studies of multilayer rare-gas films adsorbed on graphite. ${ }^{13}$

For the intraplanar Xe-Xe lattice spacing of monolayer and bilayer films, our results confirm with about a factor of 2 higher precision those of earlier LEED studies by Unguris et al. ${ }^{4}$ We have shown that the monolayer and bilayer lattice constants are indistinguishable at coexistence, being equal to within $0.005 \AA$, in agreement with the calculations of Bruch 
and co-workers. ${ }^{14,15}$ To this same degree of precision, we confirm the LEED result ${ }^{4}$ that the Xe-Xe spacing reaches that of bulk Xe at the monolayer limit of compression (bilayer onset).

This result remains a challenge for theory. The lattice constant calculated in Ref. 15 at $0 \mathrm{~K}$ for a bilayer with the more stable $A B A$ stacking is about $0.01 \AA$ larger than the bulk value, and becomes equal to the bulk value for the trilayer. Thus the film reaches the bulk lattice constant a layer earlier in the experiment than in the calculation. Since the margin of stability for the trilayer chemical potential energy with respect to that of bulk is small compared to the triple-dipole energy, this result suggests that understanding of the triple-dipole term is still incomplete. ${ }^{51}$

Our observation of intensity oscillations (analogous to RHEED oscillations) at the anti-Bragg peak of the Xe specular rod provide evidence of layerwise kinetic growth up to about eight layers on the $\operatorname{Ag}(111)$ surface, extending the upper limit of about four layers inferred from a variety of earlier experiments. ${ }^{9-11}$ Investigations of the temperature dependence of these oscillations suggest that their number may decrease at low temperatures $(\sim 33 \mathrm{~K})$ where diffusion rates are smaller. Fewer oscillations were also observed at temperatures and pressures closer to equilibrium. In addition, the number of oscillations seems to depend on the quality of the $\mathrm{Ag}(111)$ substrate in a manner which we have been unable to quantify.

Specular reflectivity experiments have allowed us to characterize the atomic density profile in a direction perpendicular to the Xe/vacuum boundary for both kinetic and quasiequilibrium growth in films from monolayer to ten-layer thicknesses. We find very sharp boundaries for monolayer and bilayer films grown at quasiequilibrium. However, for films of three layers and above, which cannot be grown at quasiequilibrium, we find a broader profile which is well described by a model of independently scattering domains having a Gaussian thickness distribution. For three-layer films the standard deviation in the mean thickness is about 0.5 layers, which increases to about two layers for a ten-layer film. We have not been able to find kinetic growth conditions which both significantly sharpen the Xe/vacuum boundary of these multilayer films and which reduce the number of $A B A$ stacking faults (see below). Thus the $Q$ dependence of the specular reflectivity confirms that the decay in the intensity oscillations with time at the anti-Bragg point of the specular rod results from a growth front spanning several layers.

Despite our evidence for roughening in thicker films, all of our Xe films grown on a $\operatorname{Ag}(111)$ substrate exhibit greater structural perfection than those grown on a graphite substrate under nominal equilibrium conditions. ${ }^{13}$ We also note that the specular reflectivity of the Xe film on a $\mathrm{Ag}(111)$ substrate is more easily investigated than on the graphite (002) surface, ${ }^{13}$ where the $\mathrm{Xe}(01 l)$ scattering sits on the wing of the graphite (002) peak.

Both the high-resolution in-plane scans as well as the nonspecular rod scans on films from bilayer thickness to $\geqslant 36$ layers are consistent with successive layers being identical in structure and mutually commensurate. The $(01 l)$ rod scans reveal two sets of film domains azimuthally separated by $60^{\circ}$ about the surface normal. Although these domains should be symmetry equivalent on a (111) surface, their un- equal population [note the intensity of the (101) and (012) peaks in Figs. 3(a) and Fig. 10] indicates that there are preferential sites on the surface for nucleation of film growth. The preferred sites may be related to the mechanism which aligns the Xe film with the substrate. Most frequently, this alignment has been attributed to the nucleation of film growth at step edges along the high-symmetry directions of the surface. ${ }^{4}$

In addition, the nonspecular rod scans provide evidence of $A B A$ stacking faults in the Xe films characteristic of an hcp structure as was found for polycrystalline ${ }^{36} \mathrm{Ar}$ films. ${ }^{12}$ The capability of growing thicker films than in this previous study has allowed demonstration that the $A B A$ faults occur mainly in the interfacial region. The lateral extent of these stacking faults may also explain the decrease in coherence length observed in radial scans near bulk condensation. ${ }^{31}$ The number of stacking faults could not be decreased by annealing of the films. A possible explanation for this is that the stacking faults are "pinned" at the $\operatorname{Ag}(111) / \mathrm{Xe}$ caused by nucleation of film growth at step edges.

Alternatively, the stacking faults near the $\mathrm{Ag}(111)$ interface may have an intrinsic origin. If the complete monolayer were slightly compressed (or expanded) relative to the bulk lattice constant (by less than our experimental resolution), then some relaxation would be required to relieve this strain and allow the first layer to lock with the second and higher layers of the film at the bulk lattice constant. The $A B A$ stacking faults might be a product of such a relaxation process and therefore be insensitive to annealing. Whether the origin of the stacking faults is extrinsic ("pinning") or intrinsic, there could be domain walls separating regions of $A B A$ and $A B C$ stacking. However, our experiments offer no direct evidence of these.

Our direct measurement of the film thickness by out-of plane scans corroborates the trilayer stability predicted by the calculations of Bruch and $\mathrm{Ni}^{15}$ These studies favored $A B A$ over $A B C$ trilayer stacks by energy differences of $<0.5$ $\mathrm{K}_{\text {atom }}{ }^{-1}$. Our nonspecular scans demonstrate that $A B C$ stacking predominates in trilayer films. However, the appreciable fraction of $A B A$ stacking faults supports a small energy difference between the two structures.

Although we observe intensity oscillations at the antiBragg point of the specular rod only up to about eight layers under the most favorable circumstances, scans along the specular rod demonstrate much thicker film growth under both kinetic and quasiequilibrium conditions. We can place a lower bound of $\sim 220 \AA$ ( 63 layers) for the maximum thickness of our Xe films on the $\operatorname{Ag}(111)$ surface. This value is limited by the out-of-plane $Q$ resolution of our diffractometer.

While our results have demonstrated layerwise growth of Xe films to higher coverages than in previous experiments, they have also shown the difficulty of maintaining structural perfection in films above bilayer thickness. This suggests some caution in interpreting the results of other experiments on multilayer rare gas films such as the trilayers used in lattice dynamics studies by inelastic He-atom scattering. ${ }^{9}$ We believe it likely that these films either did not have a perfect slablike geometry or could have had an appreciable fraction of $A B A$ stacking faults. Thus comparison with lattice dynam- 
ics calculations on perfect $A B C$ trilayer slabs may not be conclusive.

\section{ACKNOWLEDGMENTS}

We wish to thank L. W. Bruch for his comments and careful reading of the manuscript. We also thank F. Y.
Hansen, P. F. Miceli, I. K. Robinson, M. B. Webb, J. Z. Larese, H. Mo, and J. M. Phillips for helpful discussions. This work was partially supported by U.S. National Science Foundation Grant Nos. DMR-8704938, DMR-9011069, and DMR-9314235 and the U.S. Department of Energy Grant No. DE-FG02-85ER45183 of the MATRIX Participating Research Team.
*Present address: Solid State Division, Oak Ridge National Laboratory, Oak Ridge, Tennessee 37831.

†Present address: Frederick Seitz Materials Research Laboratory, 104 South Goodwin Avenue, Urbana, Illinois 61801.

†Present address: Laboratoire de Physique et de Spectroscopie Electronique-UPRESA CNRS 7014, Faculté des Sciences et Techniques, 4, rue des Frères Lumière, 68093 Mulhouse Cedex, France.

${ }^{\S}$ Present address: 5119 West Louisville Court, Columbia, Missouri 65203.

"Author to whom correspondence should be addressed.

${ }^{1}$ L. W. Bruch, M. W. Cole, and E. Zaremba, Physical Adsorption: Forces and Phenomena (Oxford University Press, Oxford, 1997).

${ }^{2}$ See, for example, G. B. Hess, in Phase Transitions in Surface Films 2, Vol. 267 of NATO Advanced Study Institute, Series B: Physics, edited by H. Taub, G. Torzo, H. J. Lauter, and S. C. Fain, Jr. (Plenum, New York, 1991), p. 357.

${ }^{3}$ P. I. Cohen, J. Unguris, and M. B. Webb, Surf. Sci. 58, 429 (1976).

${ }^{4}$ J. Unguris, L. W. Bruch, E. R. Moog and M. B. Webb, Surf. Sci. 87, 415 (1979); 109, 522 (1981).

${ }^{5}$ L. W. Bruch and J. M. Phillips, Surf. Sci. 91, 1 (1980).

${ }^{6}$ J. M. Phillips and L. W. Bruch, J. Chem. Phys. 83, 3660 (1985).

${ }^{7}$ J. M. Phillips and L. W. Bruch, Phys. Rev. Lett. 60, 1681 (1988).

${ }^{8}$ M. H. W. Chan, in Phase Transitions in Surface Films 2 (Ref. 2), p. 1.

${ }^{9}$ K. D. Gibson and S. J. Sibener, J. Chem. Phys. 88, 7862 (1988); 88, 7893 (1988); K. D. Gibson, C. Cerjan, J. C. Light, and S. J. Sibener ibid. 88, 7911 (1988).

${ }^{10}$ R. J. Behm, C. R. Brundle, and K. Wandelt, J. Chem. Phys. 85, 1061 (1986).

${ }^{11}$ X. Qian and M. Bretz, Phys. Rev. Lett. 61, 1497 (1988).

${ }^{12}$ J. Z. Larese, Q. M. Zhang, L. Passell, J. M. Hastings, J. R. Dennison, and H. Taub, Phys. Rev. B 40, 4271 (1989).

${ }^{13}$ H. Hong and R. J. Birgeneau, Z. Phys. B 77, 413 (1989).

${ }^{14}$ M. S. Wei and L. W. Bruch, J. Chem. Phys. 75, 4130 (1981).

${ }^{15}$ L. W. Bruch and X.-Z. Ni, Faraday Discuss. Chem. Soc. 80, 217 (1985).

${ }^{16}$ E. Vlieg, A. W. Denier van der Gon, J. F. van der Veen, J. E. Macdonald, and C. Norris, Phys. Rev. Lett. 61, 2241 (1988).

${ }^{17}$ P. H. Fuoss, D. W. Kisker, F. J. Lamelas, and G. B. Stephenson, Phys. Rev. Lett. 69, 2791 (1992).

${ }^{18}$ H. A. van der Vegt, H. M. van Pinxteren, M. Lohmeier, E. Vlieg, and J. M. C. Thornton, Phys. Rev. Lett. 68, 2539 (1992).

${ }^{19}$ P. A. Bennett, B. DeVries, I. K. Robinson, and P. J. Eng, Phys. Rev. Lett. 69, 2539 (1992).

${ }^{20}$ R. Feidenhans'1, Surf. Sci. Rep. 10, 105 (1989).

${ }^{21}$ J. H. Neave, B. A. Joyce, P. J. Dobson, and N. Norton, Appl. Phys. A: Solids Surf. 31, 1 (1983).

${ }^{22}$ J. Als-Nielsen, in Structure and Dynamics of Surfaces II, edited by W. Schommers and P. von Blanckenhagen (Springer-Verlag, Berlin, 1987), p. 181.

${ }^{23}$ J. R. Dennison, S.-K. Wang, P. Dai, T. Angot, H. Taub, and S. N. Ehrlich, Rev. Sci. Instrum. 63, 3835 (1992).

${ }^{24}$ S.-K. Wang, Ph.D. thesis, University of Missouri-Columbia, 1992.

${ }^{25}$ N. Greiser, G. A. Held, R. Frahm, R. L. Greener, P. M. Horn, and R. M. Suter, Phys. Rev. Lett. 59, 1706 (1987).

${ }^{26}$ P. Dai, T. Angot, S. N. Ehrlich, S.-K. Wang, and H. Taub, Phys. Rev. Lett. 72, 685 (1994).

${ }^{27}$ B. H. Bellings, D. F. Bleil, R. K. Cook, H. M. Crosswhite, H. P. R. Frederikse, R. B. Lindsay, J. B. Marion, and M. W. Zemansky, in American Institute of Physics Handbook, edited by D. E. Gray (AIP, New York, 1972).

${ }^{28}$ S.-K. Wang, P. Dai, and H. Taub, J. Appl. Crystallogr. 26, 697 (1993).

${ }^{29}$ P. Dai, Ph.D. thesis, University of Missouri-Columbia, 1993.

${ }^{30}$ Although the Xe film is believed to be grown at a constant dosing rate, the effective Xe pressure is not strictly constant during the "isobar" measurement due to the decreasing temperature.

${ }^{31}$ From an increase in the radial full width at half maximum (FWHM) of the $\mathrm{Xe}(10)$ peak in the temperature range $55<T$ $<65 \mathrm{~K}$, we infer a slightly smaller lateral coherence of $\sim 315 \AA$ near bulk condensation. The FWHM decreases in the thickest films $(T<50 \mathrm{~K})$, corresponding to an increase in the coherence length to $\sim 450 \AA$.

${ }^{32}$ P. Korpiun and E. Lüscher, in Rare Gas Solids, edited by M. L. Klein and J. A. Venables (Academic, London, 1977), Vol. 2, Chap. 12.

${ }^{33}$ Note that the Xe-Xe spacing has not been normalized to the bulk value of Ref. 32 as was done in Ref. 4. The relative precision of our Xe-Xe spacing measurements is $\pm 0.001 \AA$.

${ }^{34}$ J. M. Phillips, Langmuir 5, 571 (1989).

${ }^{35}$ B. E. Warren, X-ray Diffraction (Addison-Wesley, Reading, MA, 1964).

${ }^{36}$ I. K. Robinson, Phys. Rev. B 33, 3830 (1986).

${ }^{37}$ For simplicity, we did not consider the roughness of the Ag substrate.

${ }^{38}$ Due to the out-of-plane mosaic of the $\operatorname{Ag}(111)$ substrate, the Bragg component is spread over an angular range of $\sim 0.3^{\circ}$. The diffuse contribution to the scattering is assumed to be negligible in this simple model.

${ }^{39} \mathrm{We}$ neglect any lattice relaxation of either the Ag substrate near the $\mathrm{Xe} / \mathrm{Ag}$ interface.

${ }^{40}$ At an arbitrary point along the specular rod, the intensity will still oscillate with coverage, but the oscillations will vary in amplitude.

${ }^{41}$ E. Vlieg, Ph.D. thesis, FOM Institute, 1988.

${ }^{42}$ To simplify the convolution of the instrumental resolution function, a square resolution function of width $0.01 \AA^{-1}$ was used. It is not believed that any of our conclusions concerning the partial 
occupancy of the layers and the layer spacings would be altered by using a more accurate shape of the resolution function.

${ }^{43}$ The values of $d_{\mathrm{AgXe}}$ inferred from the specular scans depend primarily on the location of the intensity extremae. Since data were not taken at small enough values of $Q_{\perp}$ to locate the intensity minimum in the near monolayer scan [Fig. 7(a)], the value of $d_{\mathrm{AgXe}}=3.64 \AA$ is believed to be less reliable (uncertainty $> \pm 0.1$ $\AA$ ) than for scans over a larger range of $Q_{\perp}$.

${ }^{44}$ The specular reflectivity scans in Figs. $7(\mathrm{~b})$ and $7(\mathrm{~d})$ extend to lower values of $Q_{\perp}$, where a correction for the beam spilling over the sample edge is required. For these scans the width of the incident beam was taken to be a fitting parameter. This resulted in better fits to the data possibly because not all of the crystal surface was contributing to the reflected intensity.

${ }^{45}$ N. Stoner, M. A. Van Hove, S. Y. Tong, and M. B. Webb, Phys. Rev. Lett. 40, 243 (1978).

${ }^{46}$ E. Vlieg, J. F. van der Veen, S. J. Gurman, C. Norris, and J. E. Macdonald, Surf. Sci. 210, 301 (1989).

${ }^{47} \mathrm{Z}$. Wu, Ph.D. thesis, University of Missouri-Columbia, 1997.

${ }^{48}$ I. K. Robinson, Aust. J. Phys. 41, 359 (1988).

${ }^{49}$ The determination of the fraction $\beta$ of rotated domains may be more uncertain in this case because the scan does not extend to high enough $Q_{\perp}$ to include all of the second peak.

${ }^{50}$ For this relatively thick film ( $\geqslant 10$ layers), the fractional occupancy of the top layer was neglected $(\theta=0)$.

${ }^{51}$ L. W. Bruch (private communication). 